\title{
An algebraic approach to on-line signal denoising and derivatives estimation
}

\author{
Josip Kasac*, Dubravko Majetic, Danko Brezak \\ University of Zagreb, \\ Faculty of Mechanical Engineering and Naval Architecture, \\ I. Lucica 5, HR-10000 Zagreb, Croatia
}

\begin{abstract}
In this paper, a new algebraic approach to the on-line signal derivatives estimation is proposed. The proposed approach is based on the conversion of the truncated Taylor series expansion to the set of linearly independent equations regarding the signal derivatives. The nonhomogeneous parts of the obtained set of equations are convolution integrals, which can be transformed to the stable linear state-space filter realization. The proposed algebraic estimator provides stable convergence without the need for periodic re-initialization, as in the case of the conventional algebraic estimators. In contrast to the Taylor series-based tracking differentiators, the proposed estimator also provides an estimation of the arbitrary number of the higher-order signal derivatives. In addition, the tuning of the estimator parameters does not depends on the filter dimension. The efficiency of the proposed estimator is illustrated by the simulation examples and experimental results related to the monitoring of the surgical drilling process.
\end{abstract}

Keywords: Algebraic estimation; Differentiators; Signal denoising; Linear systems; Continuous-time filters

\section{Introduction}

The filtering and derivatives estimation of a measured signal is necessary for many applications, such as biomechanics [1], failure diagnostics [2], signal processing [3] or control engineering $[4,5]$, among others. Various methods have been proposed for derivatives estimation of noisy signals. Numerical differentiation using difference methods is a well-known direct approach to compute the signal derivatives [6]. However, numerical differentiation is an ill-posed problem, which means that small errors in the measurement values may lead to large errors in the derivatives estimate [7].

\footnotetext{
${ }^{*}$ Corresponding author

Email addresses: jkasac@fsb.hr (Josip Kasac), dmajetic@fsb.hr (Dubravko Majetic), dbrezak@fsb.hr (Danko Brezak)
} 
Consequently, numerical differentiation is implemented using finite difference schemes in combination with low-pass filters, which provide attenuation of the measurement noise. This approach introduces an inevitable signal time delay which is particularly harmful when the estimated derivatives are used in closed-loop feedback control systems.

Other methods, such as Kalman filtering, have been proposed as an alternative to the low-pass derivative estimation filter [8]. Given that the Kalman filtering is a model-based approach that requires a priori knowledge of the noise variance, it cannot be successfully applied to the cases where arbitrary signal with unknown internal model is measured and where the statistical properties of the noise may vary.

The high-gain differentiator proposed in $[9,10]$ provides tracking of the signal derivative in the limit case when the gains tend to infinity. Unfortunately, their sensitivity to highfrequency noise also grows by increasing the values of the gains. A second-order sliding-mode differentiator has been proposed in $[11,12]$. In this kind of differentiator, the upper bound for Lipschitz constants of the signal derivatives are needed. Although there is no chattering phenomenon in signal tracking, in the second dynamical equation a switching function exists, so that the output of derivative estimation is continuous but not smooth. Therefore, the chattering phenomenon still exists in derivative estimation.

In $[13,14]$, the tracking differentiators based on inverse Taylor series expansion [15] are introduced. The proposed methods provide limited accuracy because the estimator filters cannot be designed for the order of Taylor series expansion larger than $n=4$. For $n \leq 4$, the estimation accuracy can be enhanced by increasing the filter gain but, simultaneously, the sensitivity to the small high-frequency noise grows rapidly.

An algebraic approach to derivative estimation that is based on truncated Taylor series expansion is proposed in $[16,17]$. This approach provides the ability to compute fast and non-asymptotic derivative estimations of signal corrupted by noise, which is attenuated by low-pass filters without any precise knowledge of its statistical properties. However, one of the main disadvantages of this method is that the estimation process has to be periodically re-initialized to sustain accuracy because the estimator filter state-space realization is in unstable time-varying Brunovsky's form.

Despite the many derivative estimator design techniques and their application in the literature, there are only a few comparative performance studies of different derivative estimators $[18,19,20]$. A performance comparison of several differentiators is made in [20], which includes tracking differentiator and three variant of higher-order sliding-mode differentiators. It is shown that all differentiators perform well in the case of the derivative estimation of low frequency signal. However, in the case of a high-frequency signal, and whether it has noise or not, most of the differentiators perform poorly, except for the tracking differentiator [13].

In this paper, a new approach to on-line algebraic derivatives estimation is proposed. The proposed approach is based on the truncated Taylor series expansion, similarly to tracking $[13,14]$ and algebraic $[16,17]$ differentiators. However, in contrast to the tracking differentiators, the proposed estimator filter can be designed for arbitrary order of the of the Taylor series expansion, providing the arbitrary level of the estimation accuracy. In addition, in contrast to the algebraic differentiators, the proposed estimator filter state- 
space realization is in the stable Jordan canonical form, so that periodic re-initialization is not necessary.

The rest of this paper is organized as follows. The formulation of the derivative estimation problem is presented in Section 2. The solution for a particular choice of the impulse response functions is presented in Section 3. The continuous-time filter realization is considered in Section 4. The simulation results are presented in Section 5. The experimental results are presented in Section 6. Finally, the concluding remarks are summarized in Section 7.

\section{Problem formulation}

\subsection{A general framework for estimator design}

Let $u(t)$ be a continuous-time signal with bounded derivatives $u^{(1)}(t), u^{(2)}(t), \ldots, u^{(n-1)}(t)$, which we want to estimate. The truncated Taylor series expansion of $u(t-\tau)$ around $t$,

$$
u(t-\tau)=\sum_{j=0}^{n-1} \frac{(-1)^{j}}{j !} u^{(j)}(t) \tau^{j}+\frac{(-1)^{n}}{n !} u^{(n)}(\xi) \tau^{n}, \quad \xi \in[t-\tau, t]
$$

provides a connection between signal derivatives in time instant $t$ and signal value in a previous time $t-\tau$, where $\tau>0$. To determine the signal derivatives $u^{(i)}(t), i=0,1, \ldots, n-1$, on the right-hand side of (1), it is necessary to generate $n$ linearly independent equations, which is similar to the algebraic approach to parameter identification [21].

By multiplying the expression (1) with the linearly independent, bounded, square integrable functions $g_{i}(\tau), i=1, \ldots, n$, and integrating along $\tau$ from zero to $t$, the following set of equations is obtained

$$
\int_{0}^{t} g_{i}(\tau) u(t-\tau) d \tau=\sum_{j=0}^{n-1} \frac{(-1)^{j}}{j !} u^{(j)}(t) \int_{0}^{t} \tau^{j} g_{i}(\tau) d \tau+r_{i}(t) \equiv y_{i}(t)
$$

where the integral of the Lagrange remainder is

$$
r_{i}(t)=\frac{(-1)^{n}}{n !} \int_{0}^{t} u^{(n)}(\xi) \tau^{n} g_{i}(\tau) d \tau, \quad \xi \in[t-\tau, t] .
$$

By using the commutativity property of the convolution integral in Eq. (2), we get

$$
y_{i}(t)=\int_{0}^{t} g_{i}(t-\tau) u(\tau) d \tau
$$

for $i=1,2, \ldots, n$, so that $g_{i}(t)$ can be interpreted as the impulse response function of the continuous linear time-invariant filter.

By defining the elements of the moments matrix

$$
M_{i j}(t)=\frac{(-1)^{j-1}}{(j-1) !} \int_{0}^{t} \tau^{j-1} g_{i}(\tau) d \tau, \quad i, j=1,2, \ldots, n,
$$


the expression (2) can be rewritten as

$$
y_{i}(t)=\sum_{j=1}^{n} M_{i j}(t) u^{(j-1)}(t)+r_{i}(t) .
$$

Because the truncation errors $r_{i}(t)$ are unknown and the bounded functions with upper bounds which can be arbitrarily small for the appropriate choice of the impulse functions, the following expression

$$
y_{i}(t)=\sum_{j=1}^{n} M_{i j}(t) \hat{u}^{(j-1)}(t),
$$

will be used for the estimation of the signal derivatives $\hat{u}^{(i)}, i=0,1, \ldots, n-1$.

Expressions (6) and (7) can be rewritten in the matrix representation

$$
\mathbf{y}(t)=\mathbf{M}(t) \mathbf{u}(t)+\mathbf{r}(t), \quad \mathbf{y}(t)=\mathbf{M}(t) \hat{\mathbf{u}}(t),
$$

where $\mathbf{y}(t)=\left[\begin{array}{llll}y_{1}(t) & y_{2}(t) & \cdots & y_{n}(t)\end{array}\right]^{T}, \mathbf{u}(t)=\left[\begin{array}{llll}u(t) & u^{(1)}(t) & \cdots & u^{(n-1)}(t)\end{array}\right]^{T}, \hat{\mathbf{u}}(t)=$ $\left[\begin{array}{lllll}\hat{u}^{(0)}(t) & \hat{u}^{(1)}(t) & \cdots & \hat{u}^{(n-1)}(t)\end{array}\right]^{T}$ and $\mathbf{r}(t)=\left[\begin{array}{llll}r_{1}(t) & r_{2}(t) & \cdots & r_{n}(t)\end{array}\right]^{T}$. The elements of the moments matrix $\mathbf{M}(t)$ are defined by $(5)$.

The estimation of signal derivatives can be obtained from Eq. (8)

$$
\hat{\mathbf{u}}(t)=\mathbf{M}^{-1}(t) \mathbf{y}(t) .
$$

Expression (9) can be evaluated for some $t \geq \varepsilon>0$, because the matrix $\mathbf{M}(t)$ is singular in $t=0\left(M_{i j}(0)=0\right.$ for $\left.i, j=1,2, \ldots, n\right)$. This singularity in the origin is typical for the algebraic estimators, $[22,21]$, and can be avoided by using the following approximation

$$
\hat{\mathbf{u}}(t)=\left\{\begin{array}{l}
\mathbf{M}^{-1}(\varepsilon) \mathbf{y}(t), \quad t<\varepsilon, \\
\mathbf{M}^{-1}(t) \mathbf{y}(t), \quad t \geq \varepsilon
\end{array}\right.
$$

In another approach, instead of using the time-varying matrix $\mathbf{M}(t)$, we use the constant matrix $\overline{\mathbf{M}}=\lim _{t \rightarrow \infty} \mathbf{M}(t)$.

The estimation error, which is defined by $\tilde{\mathbf{u}}(t)=\hat{\mathbf{u}}(t)-\mathbf{u}(t)$, can be obtained from Eq. (8),

$$
\tilde{\mathbf{u}}(t)=\mathbf{M}^{-1}(t) \mathbf{r}(t)
$$

The performances of the estimator (9) depend on the choice of the impulse response functions $g_{i}(t), i=1,2, \ldots, n$. A good choice of the impulse response functions should satisfy the following criteria: a) the estimation error $\tilde{\mathbf{u}}(t)$ should decrease as dimension $n$ of the estimator increases; b) the transfer functions $G_{i}(s)=\mathcal{L}\left\{g_{i}(t)\right\}$ must have low-pass amplitudefrequency characteristics that provide attenuation of the high-frequency measurement noise; and c) matrix $\mathbf{M}(t)$ should be well-conditioned as the dimension $n$ of the estimator increase. 


\subsection{Diagonalization of the moments matrix}

The computationally most simple estimator (9) is found in the case when the matrix $\mathbf{M}(t)$ is diagonal. The impulse response functions which provide diagonalization of the matrix $\mathbf{M}(t)$ are time derivatives of the Dirac delta function

$$
g_{i}(t)=\delta^{(i-1)}(t), \quad i=1,2, \ldots, n .
$$

By using the following property of delta function [23],

$$
\tau^{n} \delta^{(n)}(\tau)=(-1)^{n} n ! \delta(\tau)
$$

the elements of the moments matrix becomes

$$
M_{i j}(t)=\frac{(-1)^{j-1}}{(j-1) !} \int_{0}^{t} \tau^{j-1} \delta^{(i-1)}(\tau) d \tau=\delta_{i j},
$$

where $\delta_{i j}$ is the Kronecker delta, so that

$$
y_{i}(t)=\sum_{j=1}^{\infty} M_{i j}(t) u^{(j-1)}(t)=\sum_{j=1}^{\infty} \delta_{i j} u^{(j-1)}(t)=u^{(i-1)}(t) .
$$

However, the transfer function $G_{i}(s)=\mathcal{L}\left\{g_{i}(t)\right\}=\mathcal{L}\left\{\delta^{(i-1)}(t)\right\}=s^{i-1}$ has a high-pass amplitude-frequency characteristic, which amplifies the high-frequency additive noise.

Although the solution (12) is not implementable, it will be a good starting point for the choice of the impulse response functions based on the appropriate approximation of the Dirac delta function. The main idea is to chose the continuous square integrable impulse response functions $g_{i}(a, n ; t)$ which depend on a free parameter $a$ and on the order of truncation error $n$, such that $\lim _{a \rightarrow \infty} g_{i}(a, n ; t) \rightarrow \delta(t)$. In this way, the accuracy of the estimator can be enhanced by increasing the values of the parameters $a$ and $n$.

\section{The estimator impulse response functions}

\subsection{A choice of the impulse response functions}

We choose the following impulse response function

$$
g_{1}(t)=\frac{a^{n}}{(n-1) !} t^{n-1} e^{-a t}
$$

and its time derivatives

$$
g_{i}(t)=\frac{d}{d t} g_{i-1}(t)=\frac{d^{i-1}}{d t^{i-1}} g_{1}(t)=g_{1}^{(i-1)}(t), \quad i=2,3, \ldots, n,
$$

where $a$ is some positive parameter and $n$ is positive integer. Now, we will show that function (15), in limit when $a \rightarrow \infty$, satisfies basic properties of the Dirac delta function [24],

$$
\int_{-\infty}^{\infty} \delta(t) d t=\int_{0_{-}}^{\infty} \delta(t) d t=1, \quad \delta(t)=\left\{\begin{aligned}
\infty, & t=0 \\
0, & t \neq 0
\end{aligned}\right.
$$


The integral of the impulse function (15) is

$$
\int_{0}^{\infty} g_{1}(t) d t=\frac{a^{n}}{(n-1) !} \int_{0}^{\infty} t^{n-1} e^{-a t} d t=1
$$

for any $n=1,2, \ldots$ and $a>0$. The maximum of the function $g_{1}(t)$ is in the time instant $t_{0}=(n-1) / a$, so that

$$
\lim _{a \rightarrow \infty} \max _{t \in \mathbb{R}_{+}} g_{1}(t)=\lim _{a \rightarrow \infty} g_{1}\left(t_{0}\right)=\lim _{a \rightarrow \infty}\left(a \frac{(n-1)^{n-1}}{(n-1) !} e^{-(n-1)}\right)=\infty,
$$

and $\lim _{a \rightarrow \infty} t_{0}=\lim _{a \rightarrow \infty} \frac{n-1}{a}=0_{+}$. Furthermore, if we introduce $t=t_{0}+\tilde{t}$, where $\tilde{t}>0$, and look for $\lim _{a \rightarrow \infty} g_{1}\left(t_{0}+\tilde{t}\right)$ for any $\tilde{t}>0$, then we get

$$
g_{1}\left(t_{0}+\tilde{t}\right)=\frac{a^{n}}{(n-1) !}\left(t_{0}+\tilde{t}\right)^{n-1} e^{-a\left(t_{0}+\tilde{t}\right)}=\frac{(n-1)^{n-1} e^{-(n-1)}}{(n-1) !} a\left(1+\frac{a}{n-1} \tilde{t}\right)^{n-1} e^{-a \tilde{t}}
$$

so that

$$
\lim _{a \rightarrow \infty} g_{1}\left(t_{0}+\tilde{t}\right)=0, \quad \forall \tilde{t}>0 .
$$

So, the function $\delta_{+}(t)=\lim _{a \rightarrow \infty} g_{1}(t)$ is very similar to Dirac delta function with difference in the property: $\delta_{+}\left(0_{+}\right)=\infty$ and $\delta_{+}(0)=0$, which is in contrast with the property of the Dirac delta function: $\delta(0)=\infty$ and $\delta\left(0_{-}\right)=0$. This property provide that the function $\delta_{+}(t)$ is contained inside of the limits of integration $[0, t]$. This is in contrast to the Dirac delta function, which is contained inside the interval $\left(0_{-}, t\right],[25]$.

\subsection{Properties of the moments matrix}

Proposition 1. The elements of the moments matrix (5) where the impulse functions $g_{i}(t)$, $i=1, \ldots, n$, are defined by (15) and (16) can be analytically computed as follows

$$
M_{i j}(t)=\bar{M}_{i j}+e^{-a t} H_{i j}(t)
$$

with

$$
\begin{gathered}
\bar{M}_{i j}=(-1)^{i+j-2} a^{i-j} \sum_{l=0}^{i-1} \gamma_{i j l}, \\
H_{i j}(t)=(-1)^{i+j-2} a^{i-j} \sum_{l=0}^{i-1} \gamma_{i j l} \sum_{k=0}^{n+j-l-2} \frac{a^{k}}{k !} \tau^{k},
\end{gathered}
$$

where

$$
\gamma_{i j l}=(-1)^{l}\left(\begin{array}{c}
i-1 \\
l
\end{array}\right)\left(\begin{array}{c}
n+j-l-2 \\
j-1
\end{array}\right)
$$


Proof. By shifting indexes $i \rightarrow i+1$ and $j \rightarrow j+1$ in expression $(5)$, where $g_{i}(t)=g_{1}^{(i-1)}(t)$, we get

$$
M_{i+1, j+1}(t)=\frac{(-1)^{j}}{j !} \int_{0}^{t} \tau^{j} g_{1}^{(i)}(\tau) d \tau
$$

The derivatives of the impulse function $g_{1}(t)=A t^{n-1} e^{-a t}$, where $A=a^{n} /(n-1)$ !, are

$$
g_{1}^{(i)}(t)=A \frac{d^{i}}{d t^{i}}\left(t^{n-1} e^{-a t}\right)=A \sum_{k=0}^{i}\left(\begin{array}{l}
i \\
k
\end{array}\right)\left(\frac{d^{i-k}}{d t^{i-k}} e^{-a t}\right)\left(\frac{d^{k}}{d t^{k}} t^{n-1}\right),
$$

where Leibnitz's product rule for higher derivatives is used. The multiple derivatives on the right-hand side of the expression (27) are

$$
\frac{d^{i-k}}{d t^{i-k}} e^{-a t}=(-1)^{i-k} a^{i-k} e^{-a t}, \quad k=0,1, \ldots, i
$$

and

$$
\frac{d^{k}}{d t^{k}} t^{n-1}=\frac{(n-1) !}{(n-k-1) !} t^{n-k-1}, \quad k=0,1, \ldots, n-1 .
$$

By inserting expressions (28) and (29) in Eq. (27), we get

$$
g_{1}^{(i)}(t)=a^{n} e^{-a t} \sum_{k=0}^{i}\left(\begin{array}{l}
i \\
k
\end{array}\right) \frac{(-1)^{i-k} a^{i-k}}{(n-k-1) !} t^{n-k-1} .
$$

By inserting Eq. (30) in Eq. (26), and using analytic solution of the following integral

$$
\int_{0}^{t} \tau^{n} e^{-a \tau} d \tau=\frac{n !}{a^{n+1}}\left(1-e^{-a t} \sum_{k=0}^{n} \frac{a^{k}}{k !} t^{k}\right)
$$

we get after some algebraic manipulations

$$
M_{i+1, j+1}(t)=(-1)^{i+j} a^{i-j} \sum_{k=0}^{i} \lambda_{i j k}\left(1-e^{-a t} \sum_{p=0}^{n+j-k-1} \frac{a^{p}}{p !} t^{p}\right),
$$

where

$$
\lambda_{i j k}=(-1)^{k}\left(\begin{array}{l}
i \\
k
\end{array}\right)\left(\begin{array}{c}
n+j-k-1 \\
j
\end{array}\right) .
$$

After rearranging the expression (32) and shifting indexes back $i \rightarrow i-1$ and $j \rightarrow j-1$ we get the final analytic solution (22)-(25).

From the analytic solution of the moments matrix (22) it follows that

$$
\lim _{t \rightarrow \infty} M_{i j}(t)=\bar{M}_{i j}
$$

since the term $e^{-a t} H_{i j}(t)$ converges to zero as $t \rightarrow \infty$. The convergence to the zero is faster as the parameter $a$ is larger. In other words, after a short transient response, the constant matrix $\overline{\mathbf{M}}$ will be good approximation of the time-varying matrix $\mathbf{M}(t)$. This provides much easier computation of the matrix inverse. 
Proposition 2. Matrix $\overline{\mathbf{M}}$ with elements $\bar{M}_{i j}$ is the upper triangular Toeplitz matrix with the property: $\bar{M}_{i j}=\bar{m}_{j-i}$ for $j \geq i$, and $\bar{M}_{i j}=0$ for $j<i$, where

$$
\bar{m}_{k}=\frac{\hat{m}_{k}}{a^{k}}, \quad \hat{m}_{k}=(-1)^{k}\left(\begin{array}{c}
n+k-1 \\
k
\end{array}\right), \quad k=1,2, \ldots, n-1 .
$$

For $k=0$ we have $\bar{m}_{k}=1$, so that matrix $\overline{\mathbf{M}}$ has the following structure

$$
\overline{\mathbf{M}}=\left[\begin{array}{cccccc}
1 & \frac{\hat{m}_{1}}{a} & \frac{\hat{m}_{2}}{a^{2}} & \frac{\hat{m}_{3}}{a^{3}} & \cdots & \frac{\hat{m}_{n-1}}{a^{n-1}} \\
0 & 1 & \frac{\hat{m}_{1}}{a} & \frac{\hat{m}_{2}}{a^{2}} & \cdots & \frac{\hat{m}_{n-2}}{a^{n-2}} \\
0 & 0 & 1 & \frac{\hat{m}_{1}}{a} & \cdots & \frac{\hat{m}_{n-3}}{a^{n-3}} \\
0 & 0 & 0 & 1 & \cdots & \frac{\hat{m}_{n-4}}{a^{n-4}} \\
\vdots & \vdots & \vdots & \vdots & \ddots & \vdots \\
0 & 0 & 0 & 0 & \cdots & 1
\end{array}\right] .
$$

Proof. By definition (34) and (22), the elements of matrix $\overline{\mathbf{M}}$ are

$$
\bar{M}_{i, j}=A_{j} \int_{0}^{\infty} \tau^{j-1} g_{1}^{(i-1)}(\tau) d \tau, \quad A_{j}=\frac{(-1)^{j-1}}{(j-1) !}
$$

where $i, j=1, \ldots, n$. By replacing the index $j=i+k$, the previous expression becomes

$$
\bar{M}_{i, i+k}=A_{i+k} \int_{0}^{\infty} \tau^{i+k-1} g_{1}^{(i-1)}(\tau) d \tau,
$$

where $i=1, \ldots, n$ and $k=-i+1,-i+2, \ldots,-i+n$. After taking $i-1$ partial integrations, where the property of the impulse functions $g_{i}(0)=g_{i}(\infty)=0$ is used, we get

$$
\bar{M}_{i, i+k}=(-1)^{i-1} A_{i+k} \int_{0}^{\infty}\left(\frac{d^{i-1}}{d \tau^{i-1}} \tau^{i+k-1}\right) g_{1}(\tau) d \tau
$$

where

$$
\frac{d^{i-1}}{d \tau^{i-1}} \tau^{i-1+k}= \begin{cases}\frac{(i-1+k) !}{k !} \tau^{k}, & k=0,1, \ldots, n-i \\ 0, & k=-1,-2, \ldots,-(i-1)\end{cases}
$$

so that

$$
\bar{M}_{i, i+k}=\frac{(-1)^{k}}{k !} \int_{0}^{\infty} \tau^{k} g_{1}(\tau) d \tau, \quad k=0,1, \ldots, n-i,
$$

and $\bar{M}_{i, i+k}=0$ for $k=-1,-2, \ldots,-(i-1)$. By inserting Eq. (15) in Eq. (41) and using (31) in limit when $t \rightarrow \infty$, we finally obtain

$$
\bar{M}_{i, i+k}=\frac{(-1)^{k}}{k !} \frac{a^{n}}{(n-1) !} \int_{0}^{\infty} \tau^{n+k-1} e^{-a \tau} d \tau=\frac{(-1)^{k}}{a^{k}}\left(\begin{array}{c}
n+k-1 \\
k
\end{array}\right)
$$

for $k=0,1, \ldots, n-i$ and $\bar{M}_{i, i+k}=0$ for $k=-1,-2, \ldots,-(i-1)$. We can see that the right-hand side of the above expression does not depends on the index $i$, which means that $\bar{M}_{i, i+k}=\bar{m}_{k}$ and the matrix $\overline{\mathbf{M}}$ is upper triangular Toeplitz matrix. 
Proposition 3. The inverse of the upper triangular Toeplitz matrix $\overline{\mathbf{M}}$, denoted as $\mathbf{W}=$ $\overline{\mathbf{M}}^{-1}$, is also the upper triangular Toeplitz matrix with the elements which satisfy $W_{i j}=w_{j-i}$ for $j \geq i$, and $W_{i j}=0$ for $j<i$, where

$$
w_{k}=\frac{1}{a^{k}} \hat{w}_{k}, \quad k=0,1,2, \ldots, n-1,
$$

with

$$
\hat{w}_{k}=-\sum_{j=1}^{k} \hat{w}_{k-j} \hat{m}_{j}, \quad \hat{w}_{0}=1, \quad k=1,2, \ldots, n-1 .
$$

Proof. The inverse of upper triangular Toeplitz matrix $\overline{\mathbf{M}}$ with the elements $\bar{M}_{i j}=\bar{m}_{j-i}$ is also the upper triangular Toeplitz matrix $\mathbf{W}=\overline{\mathbf{M}}^{-1}$ with the elements which satisfy $W_{i j}=w_{j-i}$ for $j \geq i$, and $W_{i j}=0$ for $j<i$, where

$$
w_{k}=-\sum_{j=1}^{k} w_{k-j} \bar{m}_{j}, \quad w_{0}=1, \quad k=1,2, \ldots, n-1 .
$$

(see [26]). Now, we will prove by induction that

$$
w_{k}=\frac{1}{a^{k}} \hat{w}_{k}, \quad \hat{w}_{k}=-\sum_{j=1}^{k} \hat{w}_{k-j} \hat{m}_{j}, \quad \hat{w}_{0}=1, \quad k=1,2, \ldots, n-1,
$$

where $\hat{m}_{j}=a^{j} \bar{m}_{j}$, from Eq. (35).

First, we will prove the base case when $k=1$. From Eq. (45), it follows for $k=1$,

$$
w_{1}=-\sum_{j=1}^{1} w_{1-j} \bar{m}_{j}=-\bar{m}_{1}=-\frac{\hat{m}_{1}}{a}=\frac{1}{a}\left(-\sum_{j=1}^{1} \hat{w}_{1-j} \hat{m}_{j}\right)=\frac{\hat{w}_{1}}{a},
$$

and (46) is true for $k=1$.

In inductive step, we suppose that Eq. (46) is true for some $k$ and we should prove that (46) holds for $k+1$. From Eq. (45), (35) and induction hypothesis (46) it follows

$$
w_{k+1}=-\sum_{j=1}^{k+1} w_{k+1-j} \bar{m}_{j}=-\sum_{j=1}^{k+1} \frac{\hat{w}_{k+1-j}}{a^{k+1-j}} \cdot \frac{\bar{m}_{j}}{a^{j}}=\frac{1}{a^{k+1}}\left(-\sum_{j=1}^{k+1} \hat{w}_{k+1-j} \hat{m}_{j}\right)=\frac{\hat{w}_{k+1}}{a^{k+1}} .
$$

Thus, Eq. (46) holds for $k+1$, and the proof of the induction step is complete.

In other words, matrix $\mathbf{W}$ has the same structure as matrix $\overline{\mathbf{M}}$, in the case when the coefficients $\hat{m}_{k}$ are replaced by $\hat{w}_{k}, k=1,2, \ldots, n-1$. 
Proposition 4. Let $\tilde{\mathbf{u}}(\infty)=\mathbf{W r}(\infty)$ be the asymptotic value of the estimation error defined by Eq. (11), where $\tilde{\mathbf{u}}(\infty)=\lim _{t \rightarrow \infty} \tilde{\mathbf{u}}(t)$ and $\mathbf{r}(\infty)=\lim _{t \rightarrow \infty} \mathbf{r}(t)$. The integral of truncation error $\mathbf{r}(t)$ is defined by Eq. (3) and the elements of the matrix $\mathbf{W}=\overline{\mathbf{M}}^{-1}$ are obtained from (43) and (44). Assume that the $n$-th derivative of the signal is bounded by

$$
\max _{t \geq 0}\left|u^{(n)}(t)\right| \leq K_{n}
$$

Then, the upper bounds on the estimation errors in asymptotic state of the signal derivatives are defined by the following expression

$$
\left|\tilde{u}_{i}(\infty)\right|=\lim _{t \rightarrow \infty}\left|u^{(i-1)}(t)-\hat{u}^{(i-1)}(t)\right| \leq \frac{K_{n}}{a^{n-i+1}}\left|C_{i}\right|, \quad i=1,2, \ldots, n,
$$

where

$$
C_{i}=\sum_{j=i}^{n}(-1)^{n+1-j}\left(\begin{array}{c}
2 n-j \\
n+1-j
\end{array}\right) \hat{w}_{j-i}
$$

Proof. The asymptotic error $\tilde{\mathbf{u}}(\infty)=\mathbf{W r}(\infty)$ can be estimated as

$$
\begin{aligned}
\left|\tilde{u}_{i}(\infty)\right| & =\left|\sum_{j=1}^{n} W_{i j} r_{j}(\infty)\right|=\left|\sum_{j=i}^{n} w_{j-i} r_{j}(\infty)\right| \\
& \leq \max _{\xi \in[0, \infty)}\left|u^{(n)}(\xi)\right|\left|\sum_{j=i}^{n} w_{j-i} \frac{(-1)^{n}}{n !} \int_{0}^{\infty} \tau^{n} g_{j}(\tau) d \tau\right|,
\end{aligned}
$$

where we used Eq. (3) in the case $t \rightarrow \infty$ and the Toeplitz matrix property $W_{i j}=w_{j-i}$ for $j \geq i$ and $W_{i j}=0$ for $j<i$. Further, by using expressions (5), (35), (43), (49) and property of the the Toeplitz matrix $\bar{M}_{i j}=\bar{m}_{j-i}$, the above error estimation can be continued

$$
\begin{aligned}
\left|\tilde{u}_{i}(\infty)\right| & \leq \max _{\xi \in[0, \infty)}\left|u^{(n)}(\xi)\right|\left|\sum_{j=i}^{n} w_{j-i} \bar{M}_{j, n+1}\right|=K_{n}\left|\sum_{j=i}^{n} w_{j-i} \bar{m}_{n+1-j}\right| \\
& =K_{n}\left|\sum_{j=i}^{n} \frac{\hat{w}_{j-i}}{a^{j-i}} \frac{\hat{m}_{n+1-j}}{a^{n+1-j}}\right|=\frac{K_{n}}{a^{n+1-i}}\left|\sum_{j=i}^{n} \hat{w}_{j-i} \hat{m}_{n+1-j}\right| .
\end{aligned}
$$

Finally, by inserting the expression for $\hat{m}_{n+1-j}$ from Eq. (35) in the last term of Eq. (53), the following error estimate is obtained

$$
\left|\tilde{u}_{i}(\infty)\right| \leq \frac{K_{n}}{a^{n+1-i}}\left|\sum_{j=i}^{n}(-1)^{n+1-j}\left(\begin{array}{c}
2 n-j \\
n+1-j
\end{array}\right) \hat{w}_{j-i}\right|
$$

which is equivalent with the expressions (50) and (51).

Fig. 1 shows the estimation error bounds, as defined by the right-hand side of Eq. (50), depending on the parameter $a$ and the dimension of estimator $n$. From expression (50) and Fig. 1 we can conclude that the estimation error is smaller as the parameter $a$ and the filter dimension $n$ is larger. In addition, the estimation accuracy decreases as the signal derivative order increases. 

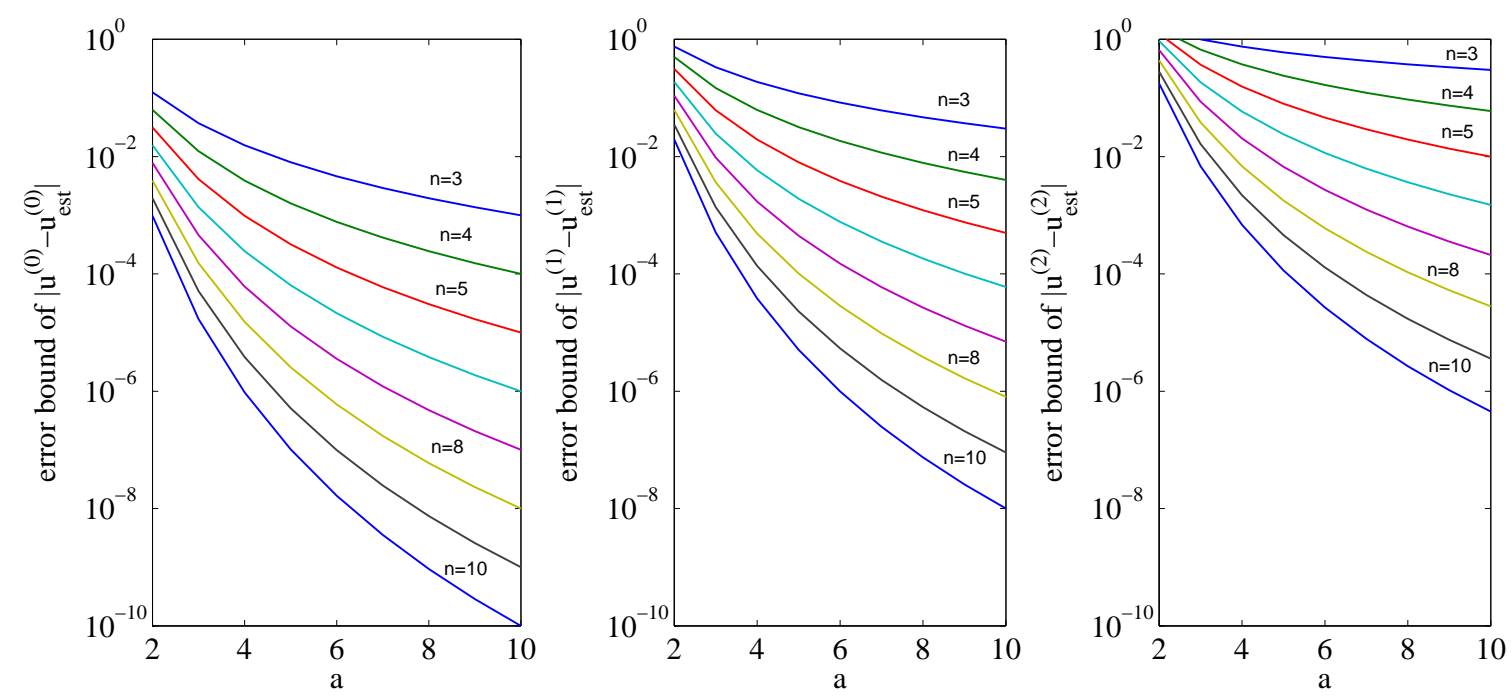

Figure 1: The upper bounds of the estimation errors for the signal $\hat{u}^{(0)}$ and derivatives $\hat{u}^{(1)}$ and $\hat{u}^{(2)}$, defined by Eq. (50), depending on the parameter $a$ and the dimension of estimator $n$.

\section{The continuous-time filter realization}

\subsection{Decomposition of the filter transfer function}

By taking the Laplace transform of the impulse response functions (15) and (16), $G_{i}^{n}(s)=$ $\mathcal{L}\left\{g_{i}(t)\right\}$ for $i=1,2, \ldots, n$, the filter transfer functions are obtained

$$
G_{1}^{n}(s)=\frac{a^{n}}{(s+a)^{n}}, \cdots G_{i}^{n}(s)=\frac{a^{n} s^{i-1}}{(s+a)^{n}}, \cdots G_{n}^{n}(s)=\frac{a^{n} s^{n-1}}{(s+a)^{n}} .
$$

Note that all transfer functions $G_{i}^{n}(s)$ in (55) have low-pass amplitude-frequency characteristics with a slope of $-(n-i+1) 20 \mathrm{~dB} / \mathrm{dec}$, and the cut-off frequency $\omega_{c}=a$.

The following properties of the transfer functions directly follow from the previous expressions

$$
G_{1}^{k}(s) G_{1}^{l}(s)=G_{1}^{k+l}(s), \quad G_{1}^{k}(s)=\left[G_{1}^{1}(s)\right]^{k} .
$$

By using these properties we can evaluate the transfer functions (55) as follows

$$
G_{2}^{n}(s)=a \frac{a^{n-1}}{(s+a)^{n-1}} \frac{s}{(s+a)}=a \frac{a^{n-1}}{(s+a)^{n-1}}\left(1-\frac{a}{s+a}\right)=a G_{1}^{n-1}(s)\left(1-G_{1}^{1}(s)\right),
$$

that is

$$
G_{2}^{n}(s)=a G_{1}^{n-1}(s)-a G_{1}^{n}(s)
$$

Similarly,

$$
G_{3}^{n}(s)=a^{2} G_{1}^{n-2}(s)\left(1-G_{1}^{1}(s)\right)^{2}=a^{2}\left(G_{1}^{n-2}(s)-2 G_{1}^{n-1}(s)+G_{1}^{n}(s)\right),
$$


and by continuing this procedure, finally we obtain

$$
G_{i}^{n}(s)=a^{i-1} G_{1}^{n-i+1}(s)\left(1-G_{1}^{1}(s)\right)^{i-1}=a^{i-1} \sum_{k=0}^{i-1}(-1)^{k}\left(\begin{array}{c}
i-1 \\
k
\end{array}\right) G_{1}^{n+k-i+1}(s),
$$

where the binomial expansion is used in the last expression. By introducing the shorthand notation

$$
\mu_{i k}=(-1)^{k} a^{i-1}\left(\begin{array}{c}
i-1 \\
k
\end{array}\right),
$$

the expression (60) becomes

$$
G_{i}^{n}(s)=\sum_{k=0}^{i-1} \mu_{i k} G_{1}^{n+k-i+1}(s)=\sum_{k=0}^{i-1} \mu_{i k}\left[G_{1}^{1}(s)\right]^{n+k-i+1} .
$$

In this way, the transfer functions (55) are represented by the serial-parallel connections of the basic transfer function $G_{1}^{1}(s)=a /(s+a)$.

The transfer function $G_{1}^{1}(s)$ is the first order low-pass filter with the cut-off frequency $\omega_{c}=a$, and the amplitude-frequency characteristic with the slope of $-20 \mathrm{~dB} / \mathrm{dec}$. In the case of a noisy signal, there will be a trade-off between the tuning rule defined by the estimation error bounds (50) and the tuning rule for the low-pass filter. By increasing parameter $a$, with the aim to improve the estimation accuracy, the cut-off frequency $\omega_{c}=a$ will also increases and this leads to a more noisy signal estimate. Meanwhile, by decreasing parameter $a$, with the aim to improve the noise attenuation properties, the estimation accuracy will also be decreased. A practical compromise to resolve the mentioned trade-off is the choice of the cut-off frequency $\omega_{c}=a$ with a value that is somewhat larger than the leading frequencies of the estimated signal.

\subsection{The state-space realization}

The input-outputs relation (4) can be expressed in complex domain as

$$
y_{i}(s)=G_{i}^{n}(s) u(s)=\sum_{k=0}^{i-1} \mu_{i k} G_{1}^{n+k-i+1}(s) u(s) .
$$

Now, we choose the following state variables

$$
\begin{gathered}
x_{1}(s)=G_{1}^{1}(s) u(s), \\
x_{2}(s)=G_{1}^{2}(s) u(s)=G_{1}^{1}(s) G_{1}^{1}(s) u(s)=G_{1}^{1}(s) x_{1}(s),
\end{gathered}
$$

and finally

$$
x_{i}(s)=G_{1}^{i}(s) u(s)=G_{1}^{1}(s) x_{i-1}(s),
$$


for $i=2,3, \ldots, n$. These expressions can be represented in time domain by the following system of linear differential equations

$$
\begin{gathered}
\dot{x}_{1}=-a x_{1}+a u(t), \\
\dot{x}_{2}=-a x_{2}+a x_{1}, \\
\vdots \\
\dot{x}_{i}=-a x_{i}+a x_{i-1}, \\
\vdots \\
\dot{x}_{n}=-a x_{n}+a x_{n-1},
\end{gathered}
$$

and output variables follows from (63)

$$
y_{i}(t)=\sum_{k=0}^{i-1} \mu_{i k} x_{n+k-i+1}(t) .
$$

The system (67)-(68) can be rewritten in the matrix state-space representation $\dot{\mathbf{x}}=\mathbf{A} \mathbf{x}+\mathbf{b} u$, $\mathbf{y}=\mathbf{C x}$, where $\mathbf{x}(t)=\left[\begin{array}{llll}x_{1}(t) & x_{2}(t) \cdots x_{n}(t)\end{array}\right]^{T}$,

$$
\mathbf{A}=\left[\begin{array}{rrrrr}
-a & 0 & 0 & \cdots & 0 \\
a & -a & 0 & \cdots & 0 \\
0 & a & -a & \cdots & 0 \\
\vdots & \vdots & \vdots & \ddots & \vdots \\
0 & 0 & 0 & \cdots & -a
\end{array}\right], \quad \mathbf{b}=\left[\begin{array}{c}
a \\
0 \\
0 \\
\vdots \\
0
\end{array}\right]
$$

or $\mathbf{A}=a \mathbf{J}_{n}^{T}(-1), \mathbf{b}=a \mathbf{e}_{1}$, where $\mathbf{e}_{1}=\left[\begin{array}{llll}1 & 0 & \cdots & 0\end{array}\right]^{T}, \mathbf{J}_{n}(-1)$ is $n \times n$ Jordan block, and

$$
\mathbf{C}=\left[\begin{array}{rrrrr}
0 & \cdots & 0 & 0 & \mu_{10} \\
0 & \cdots & 0 & \mu_{20} & \mu_{21} \\
0 & \cdots & \mu_{30} & \mu_{31} & \mu_{32} \\
\vdots & \ddots & \vdots & \vdots & \vdots \\
\mu_{n, 0} & \cdots & \mu_{n, n-3} & \mu_{n, n-2} & \mu_{n, n-1}
\end{array}\right]
$$

The final state-space realization of the estimator is

$$
\begin{aligned}
\dot{\mathbf{x}}(t) & =\mathbf{A} \mathbf{x}(t)+\mathbf{b} u(t), \quad \mathbf{x}(0)=\mathbf{0}, \\
\hat{\mathbf{u}}(t) & =\overline{\mathbf{M}}^{-1} \mathbf{C x}(t),
\end{aligned}
$$

where $\overline{\mathbf{M}}$ is defined by Eq. (34), and the elements of $\mathbf{W}=\overline{\mathbf{M}}^{-1}$ are calculated by Eq. (43) and (44). 


\subsection{Multiple filtering}

The filter (63) has a low-pass amplitude-frequency characteristic with a slope of -20 $\mathrm{dB} /$ dec. The filtered signal $\hat{u}^{(0)}(t)$, which is estimated with the best accuracy, can be used as the input of the another filter with the same structure as (71)-(72). The amplitude-frequency characteristic of such a serially connected filters has a slope of $-40 \mathrm{~dB} / \mathrm{dec}$, providing better noise attenuation properties.

Generally, in the case of the more serially connected filters, the output of the $j$-th filter is denoted as $\hat{\mathbf{u}}_{j}(t)=\left[\begin{array}{llll}\hat{u}_{j}^{(0)}(t) & \hat{u}_{j}^{(1)}(t) & \cdots & \hat{u}_{j}^{(n-1)}(t)\end{array}\right]^{T}$, where $\hat{u}_{j}^{(k)}(t)$ is the $k$-th derivative estimate after $j$-th filtering. The input of the $(j+1)$-th filter in the series is $\hat{u}_{j}^{(0)}(t)=$ $\mathbf{e}_{1}^{T} \hat{\mathbf{u}}_{j}(t)=\mathbf{e}_{1}^{T} \mathbf{W C} \mathbf{x}_{j}(t)$, where $\mathbf{x}_{j}(t)$ is the state vector of the $j$-th filter.

Finally, the serially connected filters can be represented by the following linear system

$$
\begin{aligned}
\dot{\mathbf{x}}_{1} & =-\mathbf{A} \mathbf{x}_{1}+a \mathbf{e}_{1} u(t), \\
\dot{\mathbf{x}}_{2} & =-\mathbf{A} \mathbf{x}_{2}+a \mathbf{e}_{1} \mathbf{e}_{1}^{T} \mathbf{W} \mathbf{C} \mathbf{x}_{1}, \\
& \vdots \\
\dot{\mathbf{x}}_{k} & =-\mathbf{A} \mathbf{x}_{k}+a \mathbf{e}_{1} \mathbf{e}_{1}^{T} \mathbf{W} \mathbf{C} \mathbf{x}_{k-1},
\end{aligned}
$$

and output variables

$$
\hat{\mathbf{u}}_{j}(t)=\mathbf{W C x}_{j}(t), \quad j=1,2, \ldots, k,
$$

where the transfer function between input signal $u(t)$ and estimate $\hat{\mathbf{u}}_{j}(t)$ has the low-pass amplitude-frequency characteristic with the slope of $-20 \cdot j \mathrm{~dB} / \mathrm{dec}$.

\section{Simulation examples}

The performance of the proposed estimator will be illustrated in an example, considered in $[13,20]$, related to the denoising and derivatives estimation of the signal $u(t)=\sin (\omega t)+$ $a_{n} \xi(t)$, where $\xi(t)$ is some Gauss noise of standard normal distribution $\mathcal{N}(0,1), a_{n}$ is the noise amplitude and $\omega$ is the frequency of the periodic signal. In the case without noise, the upper bounds on the estimation errors (50) are $\left|\tilde{u}^{(i)}(\infty)\right| \leq(\omega / a)^{n} a^{i}\left|C_{i+1}\right|$ for $i=$ $0,1,2, \ldots, n-1$. The necessary conditions that should be satisfied if we want that the estimation error decreases when the filter dimension $n$ increases, is $a>\omega$. In the case with noise, the value of the parameter $a$ should not be much higher than $\omega$, because the parameter $a$ is the cut-off frequency of the low-pass filter (63).

Fig. 2 shows signal $u(t)=\sin (2 t)$ and the first five derivatives estimations in the case without noise for the proposed algebraic estimator and Fig. 3 shows the estimation errors. The dashed lines in Fig. 3 are estimation error bounds obtained by Eq. (50), where $K_{n}=2^{n}$. The parameters of the algebraic estimator are $a=10$ and $n=12$. It can be seen that, after some transient time, all of the estimation errors are below the bounds defined by Eq. (50). A consequence of placing poles far in the left-half plane to provide high estimation accuracy is an impulsive-like transient behavior known as the peaking phenomenon [27, 28]. This transient behavior can be avoided with a similar approach as in the case of the algebraic parameter estimation $[22,21]$, by the evaluation of the estimates starting at a later time $t \geq t_{0}>0$. 

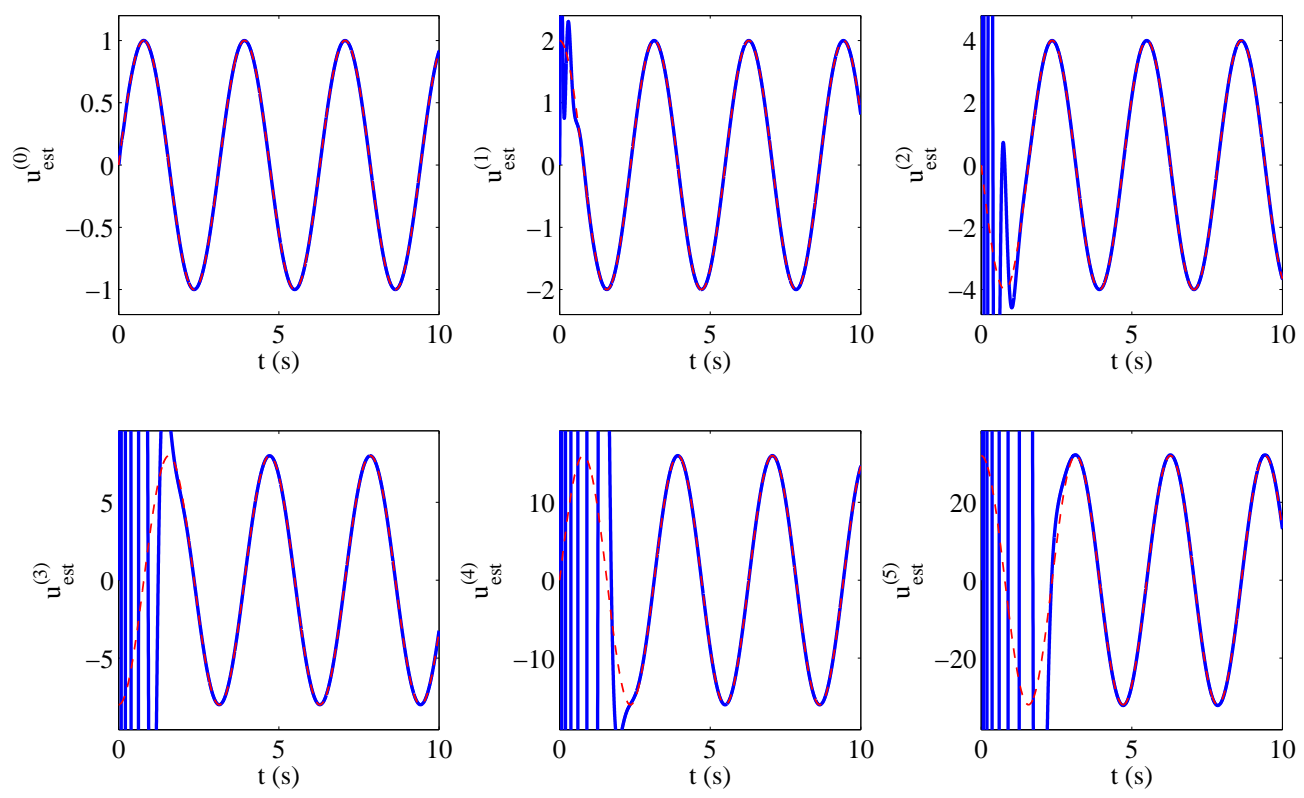

Figure 2: The estimated signal and the first five estimated derivatives for the algebraic estimator in the case without noise. The dashed lines are analytic solutions.

Given that the algebraic estimator provides the best accuracy and the best noise attenuation properties in the case of the estimation of the signal $\hat{u}^{(0)}(t)$, the denoising aspect appears as a specially interesting feature of the proposed estimator. Fig. 4 illustrates the denoising properties of the algebraic estimator in the case of the low signal-to-noise ratio, for the 1st, 2nd and 3rd filtering. The input signal is $u(t)=\sin (2 t)+0.5 \xi(t)$, where $\xi(t)$ is some Gauss noise of standard normal distribution $\mathcal{N}(0,1)$. The parameters of the algebraic estimator are $a=5$ and $n=6$. It can be seen that every additional signal filtering provides a smoother signal estimation.

\subsection{Comparison with the tracking differentiator (TD)}

The performances of the proposed algebraic differentiator (AD) will be compared with the tracking differentiator (TD) proposed in [13], which is also based on the Taylor series expansion and which have demonstrated good performance in comparison with other differentiators [20].

The third-order TD is represented by

$$
\begin{aligned}
& \dddot{r}+\frac{3}{\varepsilon} \ddot{r}+\frac{6}{\varepsilon^{2}} \dot{r}+\frac{6}{\varepsilon^{3}} r=\frac{6}{\varepsilon^{3}} u(t), \\
& \hat{u}^{(1)}(t)=\frac{3}{\varepsilon} u(t)-\frac{3}{\varepsilon} r(t)-2 \dot{r}(t)-\frac{\varepsilon}{2} \ddot{r}(t), \\
& \hat{u}^{(2)}(t)=\frac{6}{\varepsilon^{2}} u(t)-\frac{6}{\varepsilon^{2}} r(t)-\frac{6}{\varepsilon} \dot{r}(t)-2 \ddot{r}(t), \\
& 15
\end{aligned}
$$



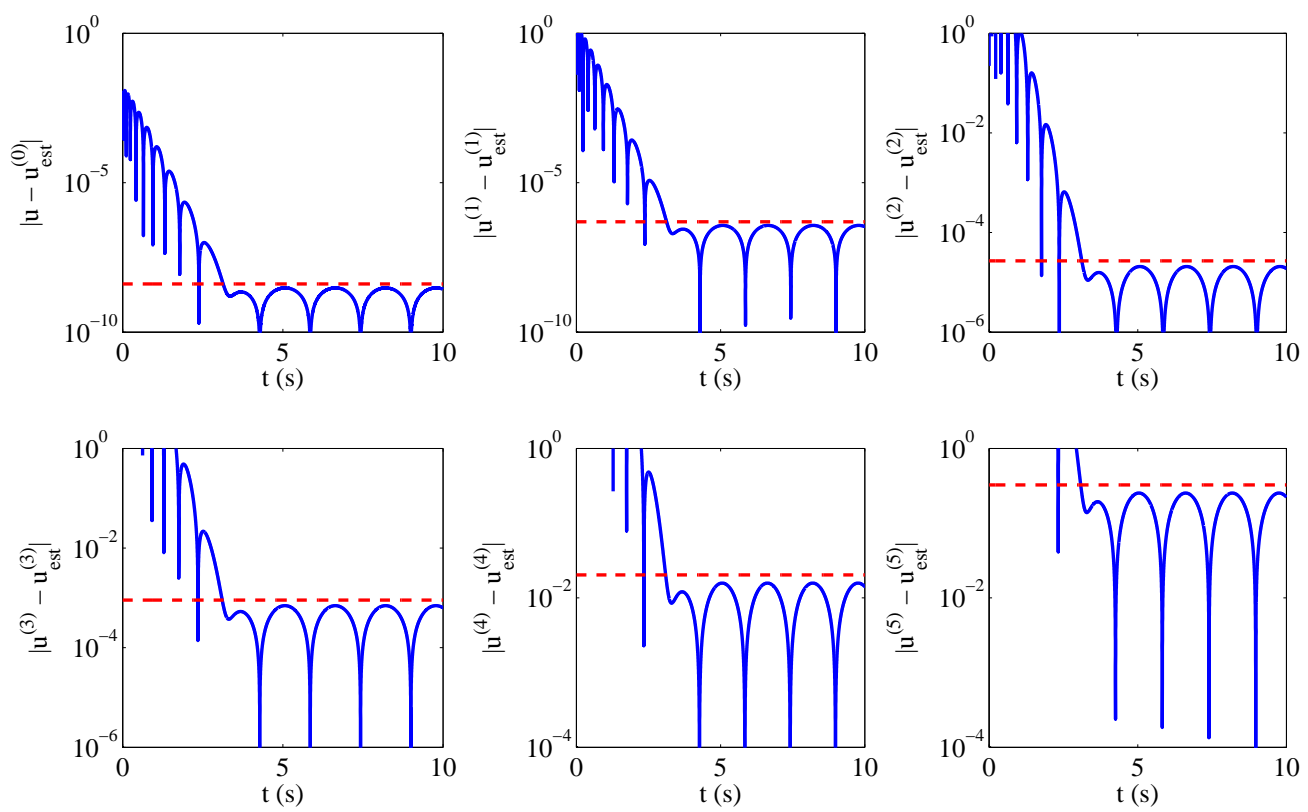

Figure 3: The estimation errors of the signal and the first five derivatives for the algebraic estimator in the case without noise. The dashed lines are bounds of the estimation errors defined by Eq. (50).
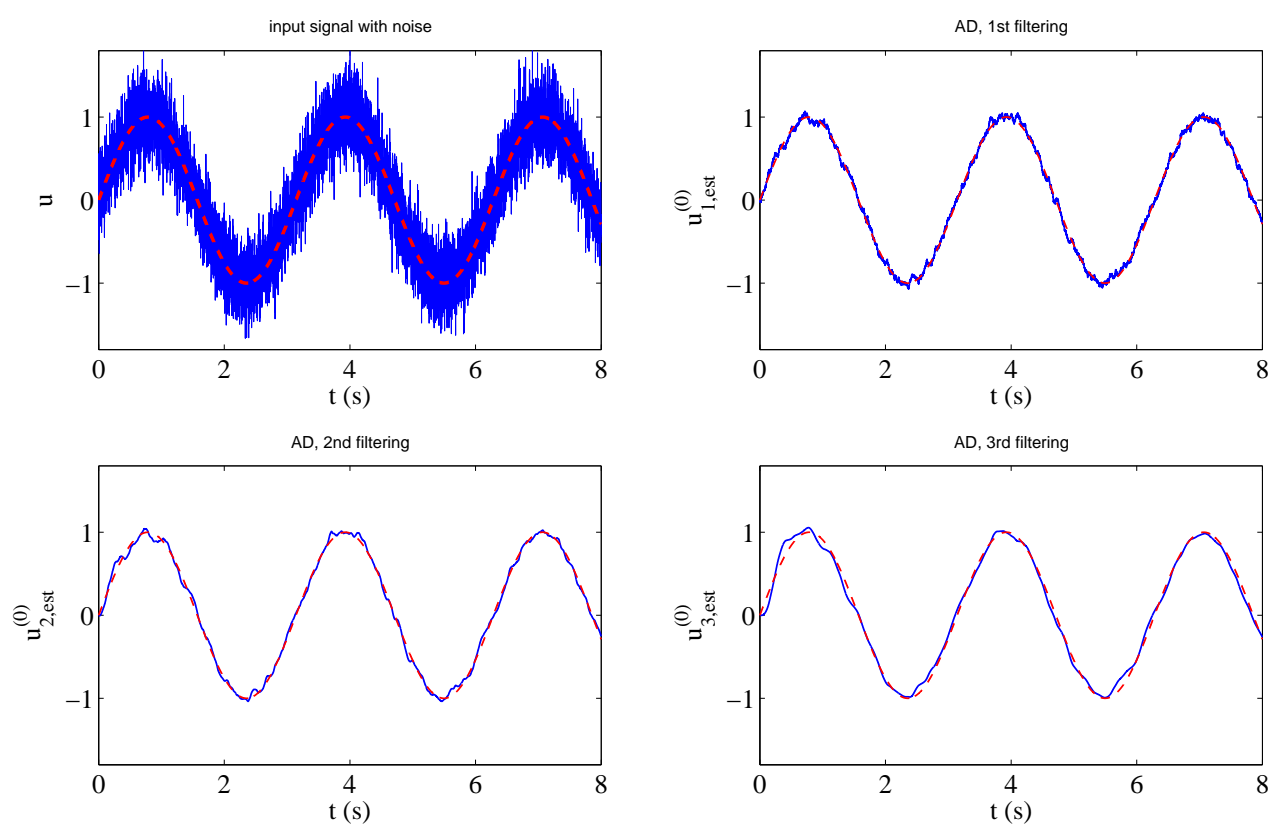

Figure 4: The denoising of the signal with the high noise amplitude by using the algebraic differentiator (AD) with 1st, 2nd and 3rd filtering. 

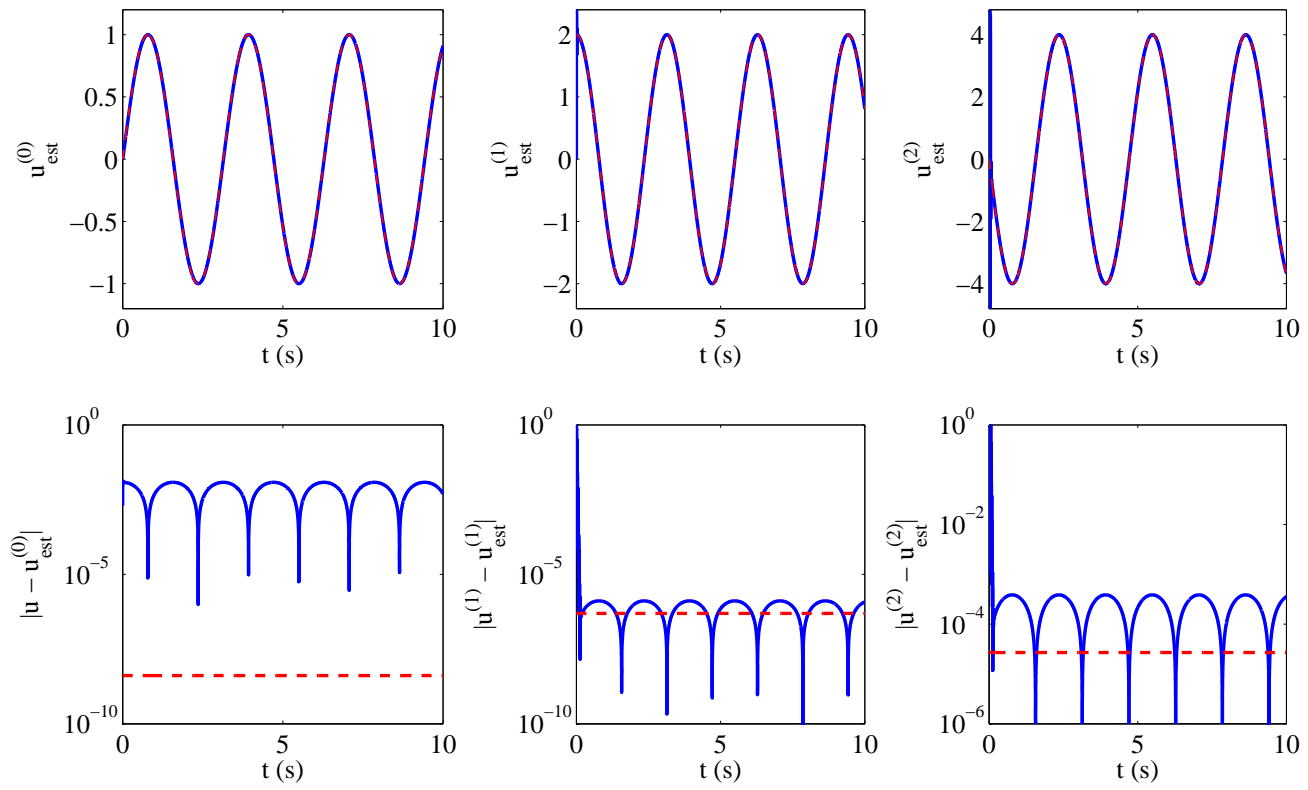

Figure 5: The estimated derivatives and estimation errors for the tracking differentiator (TD) [13] in the case without noise. The dashed lines are the same error bounds as in Fig. 3.
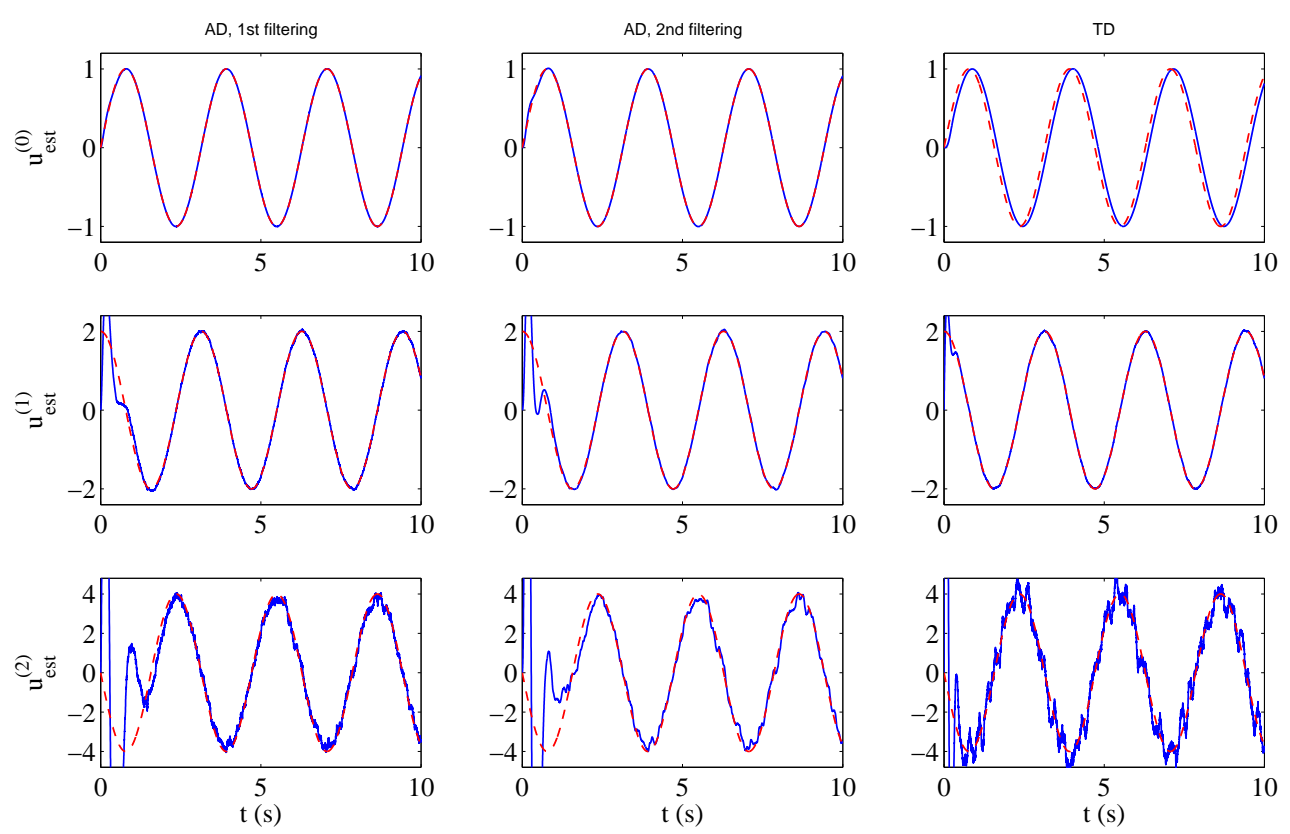

Figure 6: The estimated signals and and derivatives for the AD with 1st and 2nd filtering and for the TD [13], in the case with noise. The dashed lines are analytic solutions. 
while signal $u(t)$ is estimated by $\hat{u}^{(0)}(t)=r(t+\varepsilon)$, where $\varepsilon$ is a small positive parameter.

Fig. 5 shows the signal $u(t)=\sin (2 t)$ and the first two derivatives estimations in the case without noise for the TD. Note that TD cannot estimate higher-order derivatives. The gain parameter of the TD is chosen as $\varepsilon=0.005$ to provide the similar level of accuracy for the estimation of the first derivative $\hat{u}^{(1)}(t)$, as the algebraic estimator. The dashed lines are the same error bounds as in Fig. 3 for the algebraic estimator. It can be seen that for a similar level of accuracy of the first derivative estimation $\hat{u}^{(1)}(t)$, the algebraic estimator provides better accuracy of the second derivative estimate $\hat{u}^{(2)}(t)$ and much better accuracy of the signal $\hat{u}^{(0)}(t)$.

Fig. 6 shows estimations of the first two derivatives of the noisy signal for the algebraic estimator with the 1st and 2nd filtering, and the TD. The input signal is $u(t)=\sin (2 t)+$ $0.01 \xi(t)$, where $\xi(t)$ is some Gauss noise of standard normal distribution $\mathcal{N}(0,1)$. The parameters of the algebraic estimator are $a=5$ and $n=8$, and the parameter of the TD is $\varepsilon=0.1$, as in [13]. It can be seen that algebraic estimator provide better noise attenuation for the second derivative estimate.

Note that the accuracy of the TD in the case without noise depends on high gains $3 / \varepsilon$, $6 / \varepsilon^{2}, 6 / \varepsilon^{3}$ in Eq. (75). In addition, the estimated derivatives $\hat{u}^{(1)}(t)$ and $\hat{u}^{(2)}(t)$ depend directly on the input signals via terms $\frac{3}{\varepsilon} u(t)$ and $\frac{6}{\varepsilon^{2}} u(t)$, respectively. In the case with noise, the gain parameter $\varepsilon=0.005$, which is used for the case without noise in Fig. 5, will significantly amplify the input noise. This is the reason why the gain parameter is changed to $\varepsilon=0.1$ in the case with noise. The parameters of the algebraic estimator are also changed in the case with noise to provide better noise attenuation. However, while the parameters of the algebraic estimator are changed by a rate of approximately two times, the parameter of the TD is changed by a rate of 20 times. In other words, in comparison to the TD, the parameter tuning of the algebraic estimator is less sensitive to the stochastic characteristics of the input signal.

\subsection{Comparison with the overlapping algebraic derivatives estimator (OADE)}

In this subsection, the performance of the proposed AD will be compared with the online signal filtering algorithm presented in [17, 29]. The proposed filter, which is based on algebraic parameter and state estimation techniques [22], is composed of two subsystems: (i) an estimation scheme for the time derivatives of the measured signal $u(t)$, which is based on an overlapping implementation of the algebraic approach for time derivative estimation; and (ii) an overlapping implementation of the algebraic filtering estimation in which the input to the algebraic filter estimator is the output of the overlapping derivative estimator.

The estimates of the first three derivatives, $\hat{u}^{(j)}(t)$ for $j=1,2,3$, of a smooth signal affected by an additive noise are given by

$$
\hat{u}^{(j)}(t)= \begin{cases}\hat{u}_{2}^{(j)}(t), & \left(0 \leq t \bmod T_{r}<T_{r} / 2\right) \operatorname{AND}(t \geq \epsilon), \\ \hat{u}_{1}^{(j)}(t), & \left(T_{r} / 2 \leq t \bmod T_{r}<T_{r}\right) \text { AND }(t \geq \epsilon)\end{cases}
$$



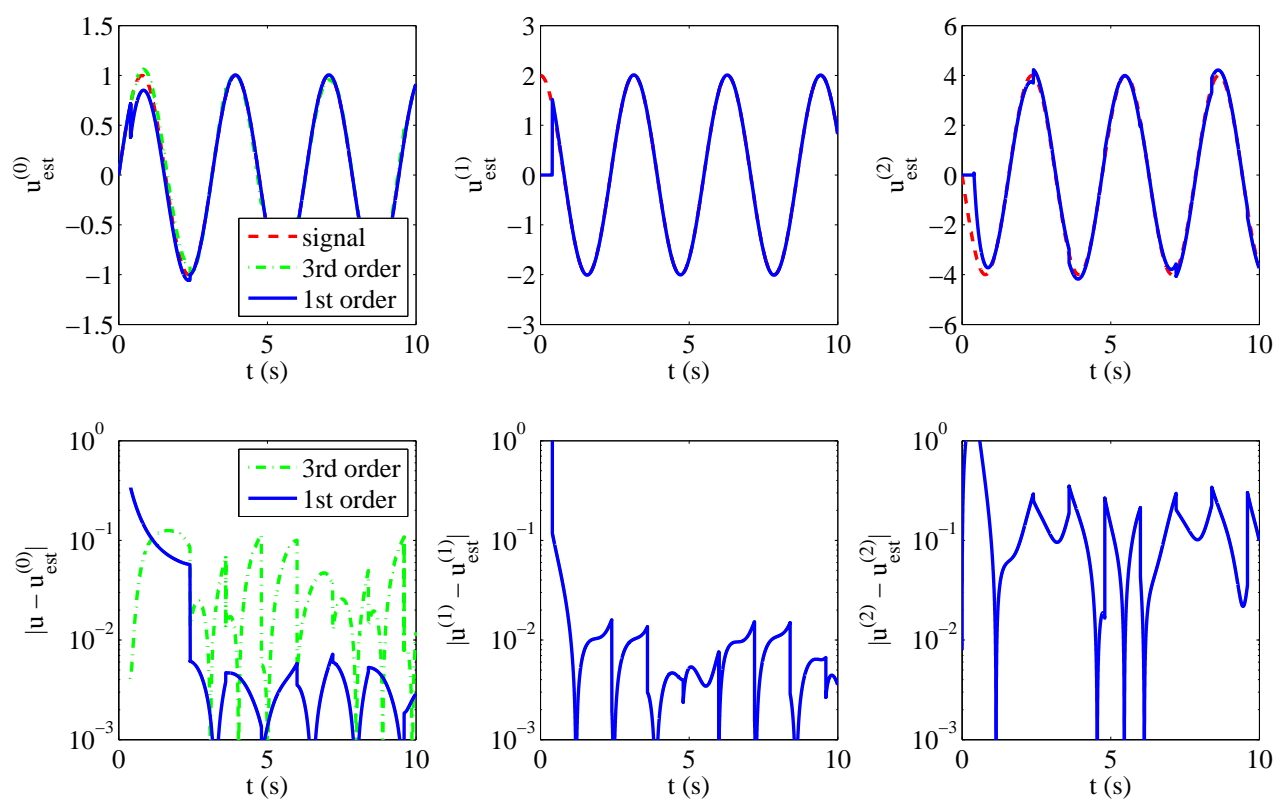

Figure 7: The estimated derivatives and estimation errors for the OADE [17] with the 1st and 3rd order filtering, in the case without noise.

where

$$
\begin{aligned}
& \hat{u}_{i}^{(1)}(t)= \begin{cases}\frac{1}{t_{i}^{7}}\left[42 t_{i}^{6} u(t)+z_{i 1}(t)\right], & \text { for } t_{i} \geq \epsilon, \\
\hat{u}_{i}^{(1)}\left(t_{i}^{-}\right), & \text {for } 0 \leq t_{i}<\epsilon,\end{cases} \\
& \hat{u}_{i}^{(2)}(t)= \begin{cases}\frac{1}{t_{i}^{8}}\left[840 t_{i}^{6} u(t)+35 z_{i 1}(t)+t_{i} z_{i 2}(t)\right], & \text { for } t_{i} \geq \epsilon, \\
\hat{u}_{i}^{(2)}\left(t_{i}^{-}\right), & \text {for } 0 \leq t_{i}<\epsilon,\end{cases} \\
& \hat{u}_{i}^{(3)}(t)= \begin{cases}\frac{1}{t_{i}^{9}}\left[10080 t_{i}^{6} u(t)+560 z_{i 1}(t)+28 t_{i} z_{i 2}(t)+t_{i}^{2} z_{i 3}(t)\right], & \text { for } t_{i} \geq \epsilon, \\
\hat{u}_{i}^{(3)}\left(t_{i}^{-}\right), & \text {for } 0 \leq t_{i}<\epsilon,\end{cases}
\end{aligned}
$$

and the corresponding filter equations are

$$
\begin{array}{ll}
\dot{z}_{i 1}=-882 t_{i}^{5} u(t)+z_{i 2}, & \dot{z}_{i 4}=52920 t_{i}^{2} u(t)+z_{i 5}, \\
\dot{z}_{i 2}=7350 t_{i}^{4} u(t)+z_{i 3}, & \dot{z}_{i 5}=-35280 t_{i} u(t)+z_{i 6}, \\
\dot{z}_{i 3}=-29400 t_{i}^{3} u(t)+z_{i 4}, & \dot{z}_{i 6}=5040 u(t),
\end{array}
$$



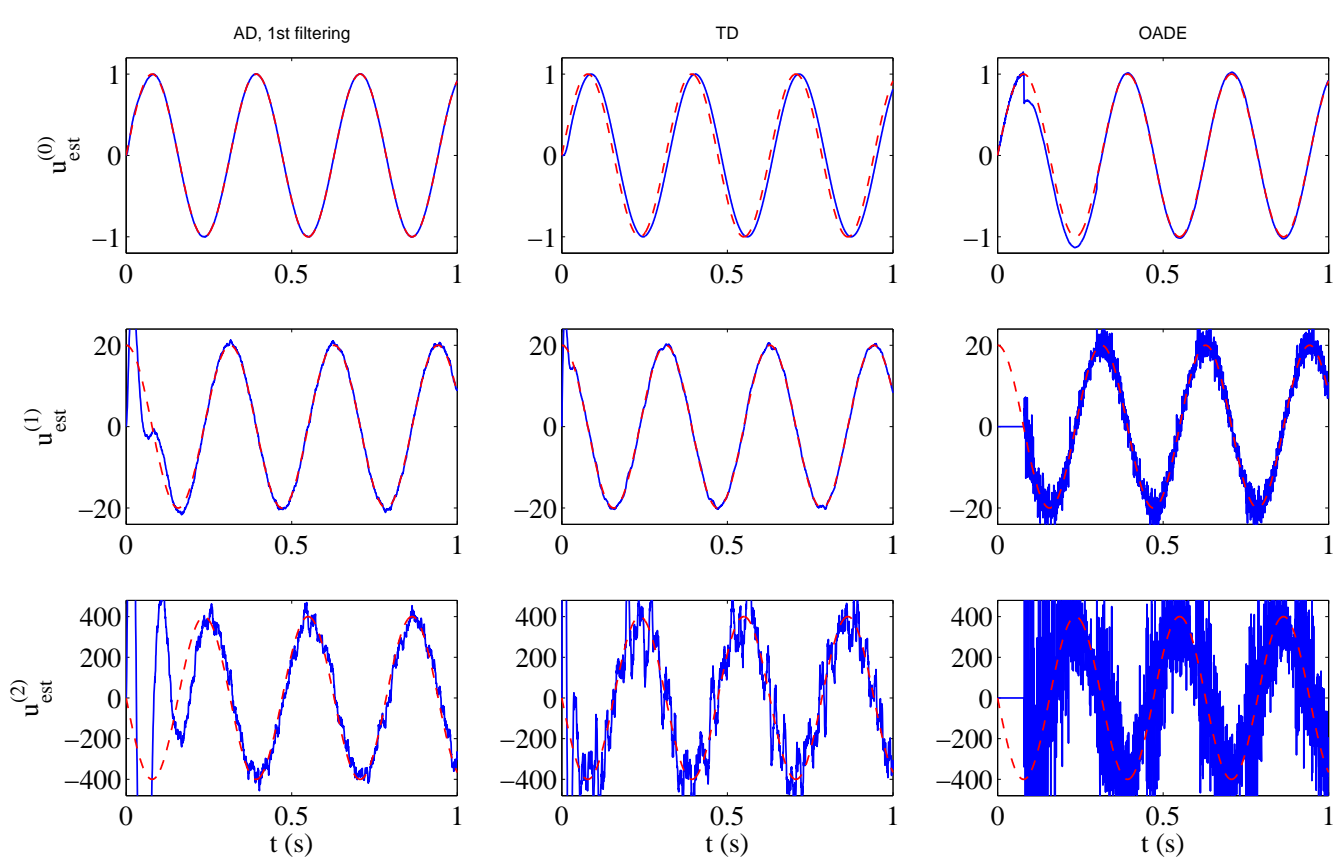

Figure 8: The estimated signals and derivatives for the proposed AD, TD [13] and OADE [17] with the 1st order signal filtering in the case with noise.

for $i=1,2$, where $T_{r}$ is the reset time, and two time lines, $t_{1}$ and $t_{2}$, are defined as follows

$$
t_{1}=t \bmod T_{r}, \quad t_{2}=\left(t-T_{r} / 2\right) \bmod T_{r} .
$$

Two estimators run simultaneously but in an overlapping fashion to obtain valid results at all times except in the initial short time interval $0 \leq t<\epsilon$. During this time interval, the zero values of derivative estimates are used. During the time interval $0 \leq t_{i}<\epsilon$, the last computed values before resetting, denoted as $\hat{u}_{i}^{(j)}\left(t_{i}^{-}\right)$for $j=1,2,3$ and $i=1,2$, are used instead of an arbitrary constant [29].

The re-initialization of each estimator is separated by a time interval of duration $T_{r} / 2$. The first estimator $(i=1)$ is re-initialized when $t_{1}=0$ and the second estimator $(i=2)$ is re-initialized when $t_{2}=0$. The proposed technique resets one of the estimators while the original remains active, and vice versa. This allows the avoidance of burst noises owing to discontinuities induced in the states of the estimator when these states are re-initialized. This policy is called the switched overlapping estimators technique.

In a similar way to the time derivative estimator, the algebraic signal filtering algorithm is obtained as follows

$$
\hat{u}_{i}^{(0)}(t)= \begin{cases}\frac{1}{t_{i}^{3}} w_{i 3}, \quad \text { for } t_{i} \geq \epsilon, & \dot{w}_{i 1}=t_{i}^{3} \hat{u}^{(3)}(t)+6 u(t), \\ \hat{u}_{i}^{(0)}\left(t_{i}^{-}\right), \text {for } 0 \leq t_{i}<\epsilon, & \dot{w}_{i 2}=-18 t_{i} u(t)+w_{i 1}\end{cases}
$$


where the final signal estimation $\hat{u}^{(0)}(t)$ is obtained by Eq. (76) for $j=0$ and $i=1,2$. During the time interval $[0, \epsilon)$, in which the results of the algebraic signal filtering is not yet valid, the original noisy signal $u(t)$ is used instead of an arbitrary constant. The use of the overlapping signal filtering makes it possible to re-initialize without singularities and it substantially improves the accuracy of the estimation of the original signal.

This is the 3rd-order algebraic signal filtering algorithm since the filter input is the third derivative estimate $\hat{u}^{(3)}(t)$. Even better accuracy of the signal estimation can be obtained by the 1st-order algebraic signal filtering algorithm

$$
\hat{u}_{i}^{(0)}(t)=\left\{\begin{array}{l}
\frac{1}{t_{i}} x_{i}, \quad \text { for } t_{i} \geq \epsilon, \\
\hat{u}_{i}^{(0)}\left(t_{i}^{-}\right), \text {for } \quad 0 \leq t_{i}<\epsilon,
\end{array} \quad \dot{x}_{i}=t_{i} \hat{u}^{(1)}(t)+u(t),\right.
$$

because the first derivative is estimated with the better accuracy than the third derivative.

Fig. 7 shows the signal and the first two derivatives estimations in the case of the input signal $u(t)=\sin (2 t)$ without noise, for the OADE. The reset time is chosen as $T_{r}=2.4$ and $\epsilon=0.4$ after several trial and error simulations to provide the best possible accuracy of the signal and derivatives estimations. As can be seen, the OADE has the lower derivatives estimation accuracy than the AD and TD but the estimated signal for the 1st order filtering has somewhat better accuracy than in the case of the TD. The estimation accuracy of the OADE can not be significantly improved by tuning the parameter $T_{r}$. Meanwhile, the OADE has no large transients as in the case of the AD and TD.

Fig. 8 shows estimations of the first two derivatives of the noisy signal for the proposed $\mathrm{AD}$, the TD and OADE. In this case, the input signal $u(t)=\sin (20 t)+0.01 \xi(t)$ has 10 times higher angular frequency then in the previous examples. The parameters of the algebraic estimator are $a=35$ and $n=10$, the parameter of the TD is $\varepsilon=0.01$, and the reset time of the OADE is $T_{r}=0.3$ and $\epsilon=0.08$. It can be seen that algebraic estimator provides better noise attenuation for the second derivative estimate. It can also be seen that the OADE has lower noise attenuation performance in the derivatives estimation than the AD and TD but the estimated signal is not delayed as in the case of the TD.

\section{Experimental results}

The experimental part of this study analyzed the application of the proposed estimator in the monitoring of the surgical drilling process. The modern medical drilling systems that are used in bone and joint surgery are characterized by a relatively low level of automation (i.e., with no process monitoring and/or adaptive control characteristics), which prevents mechanical and thermal bone damage. However, the quality of the drilling process depends solely on the operator's skills and the tool's characteristics (especially the tool's quality and condition of the cutting edges).

A review of the currently available scientific papers and patents reveals the appearance of the first solutions for advanced medical drills with integrated sensors, in the form of concept design or concrete patented types [30]. These systems combine force/torque sensors, and 


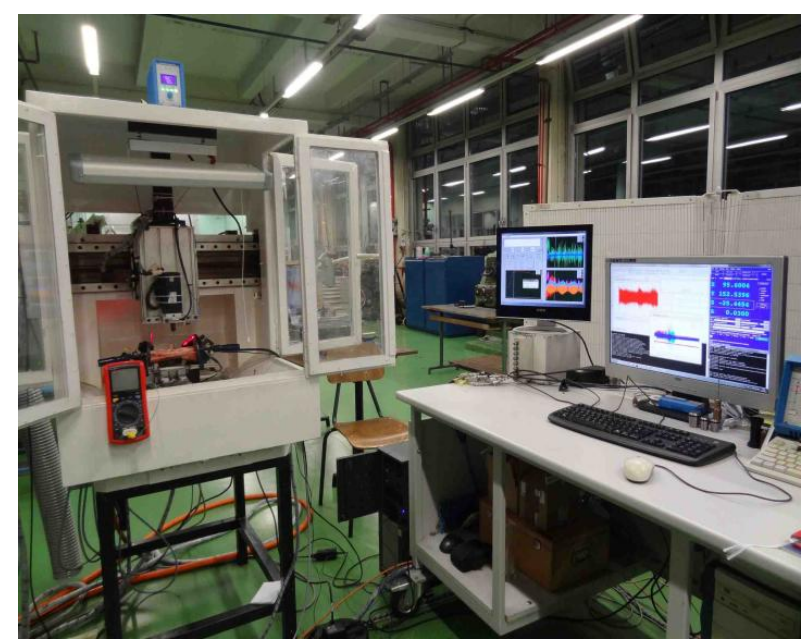

1) Acoustic emission sensor

2) Surgical drill

3) Bone specimen

4) Main spindle motor

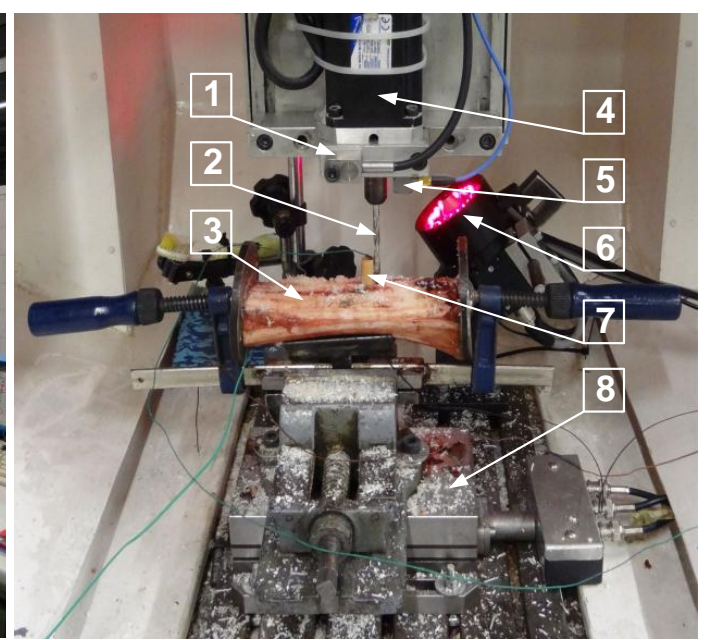

5) Accelerometer

6) Industrial CCD camera with telecentric lens system

7) Temperature probe with thermocouple

8) Force sensor

Figure 9: The experimental setup.

vibration and acceleration sensors to identify drilling depth (i.e., drilling time and feed), and also the transition from one material into another.

An important feature of the next-generation of surgical drills is the development of an automated feed movement system, which would allow the application of high-speed machining and reduce human influence in the drill's guidance. The operator would only define the drilling location and the desired tool direction/angle, all of the other activities would be performed autonomously by the machine monitoring and control system.

One of the crucial and necessary features for a semi-automated surgical drilling system is its ability to identify the beginning of the cutting process and, more important, the precise moment when the drill cutting edges exit the bone. This feature is vitally important in the reduction of the soft tissue damages located in the drill exit zone.

\subsection{The experimental setup and measurement results}

To obtain drilling process signals, an experiment was performed using surgical drill 4.5 $\mathrm{mm}$ in diameter, with a cutting speed $30 \mathrm{~m} / \mathrm{min}$, and the feed rate $0.03 \mathrm{~mm} / \mathrm{rev}$ and fresh bovine tibia with average diaphysis cortical thickness (drilling depth) of $8.5 \mathrm{~mm}$. Drilling was performed using the 3-axis bench-top mini milling machine (Fig. 9), which was adjusted for the purpose of bone drilling research [31]. This machine has been retrofitted with the $0.4 \mathrm{~kW}(1.27 \mathrm{Nm})$ permanent magnet synchronous motors with integrated incremental encoders (type Mecapion SB04A), corresponding motor controllers (DPCANIE-030A400 and DPCANIE-060A400), ball screw assemblies, and a LinuxCNC open architecture control (OAC) system. 

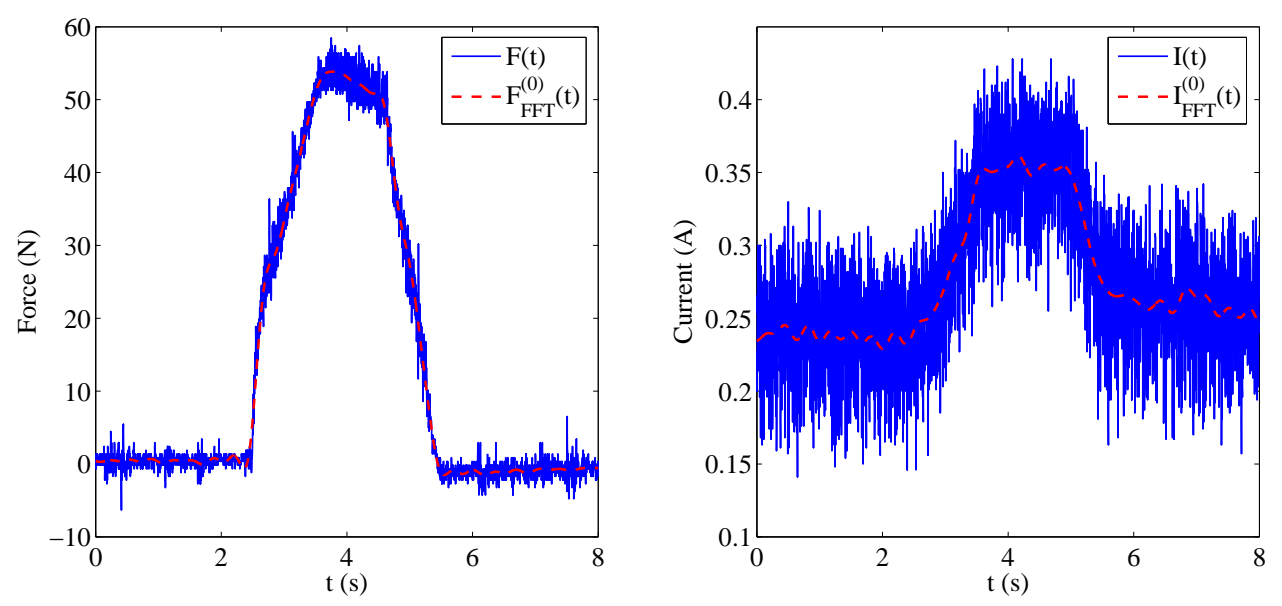

Figure 10: The measured signal for the force $F(t)$ and electric current $I(t)$. The dashed lines are filtered signals obtained by using the off-line FFT filter.

Two types of signals were measured during the drilling process: the main spindle current $I(t)$ was sampled from the motor spindle controller and the axial cutting force $F(t)$ was measured using Kistler piezoelectric dynamometer 9257B and 5017B charge amplifier. All of the signals were measured with a $1 \mathrm{~ms}$ sampling rate. The main challenge was the reliable detection of the drill exit in real time, based on highly noised measurement signal of the electric current (if possible, force signals should be replaced with the current signals to reduce the price of the system and to simplify machine handling in surgical conditions).

Fig. 10 shows the measured signal for the force $F(t)$ and electric current $I(t)$. The dashed lines are filtered signals obtained by using the off-line FFT filter with the cut-off frequency $\omega_{c}=3 \mathrm{~Hz}$. The measurements of force and current signals started while the drill bit was moving towards the bone specimen at a distance of approximately 2-3 $\mathrm{mm}$ away from the hole entrance position. When the chisel point of the drill touched the cortical bone surface, the value of the force/current signals started to increase. The values reached a maximum at the moment when both cutting edges fully entered into the bone. The values of these two signals then remained high up to the moment when chisel point broke through the cortical bone, after which they started to decrease gradually until both cutting edges had fully exited the bone.

We define the following switching function

$$
R\left(u^{(0)}(t), u^{(1)}(t)\right)= \begin{cases}1 ; & \left(u_{m} \leq u^{(0)}(t) \leq u_{M}\right) \text { AND }\left(u^{(1)}(t)<0\right) \\ 0 ; & \text { elsewhere }\end{cases}
$$

where $u^{(0)}(t)$ is the estimation of the measured signal, $u^{(1)}(t)$ is the estimation of the signal first derivative, $u_{M}$ is the defined constant below the maximal value of the signal, and $u_{m}$ is the defined constant above the nominal value of the signal. 

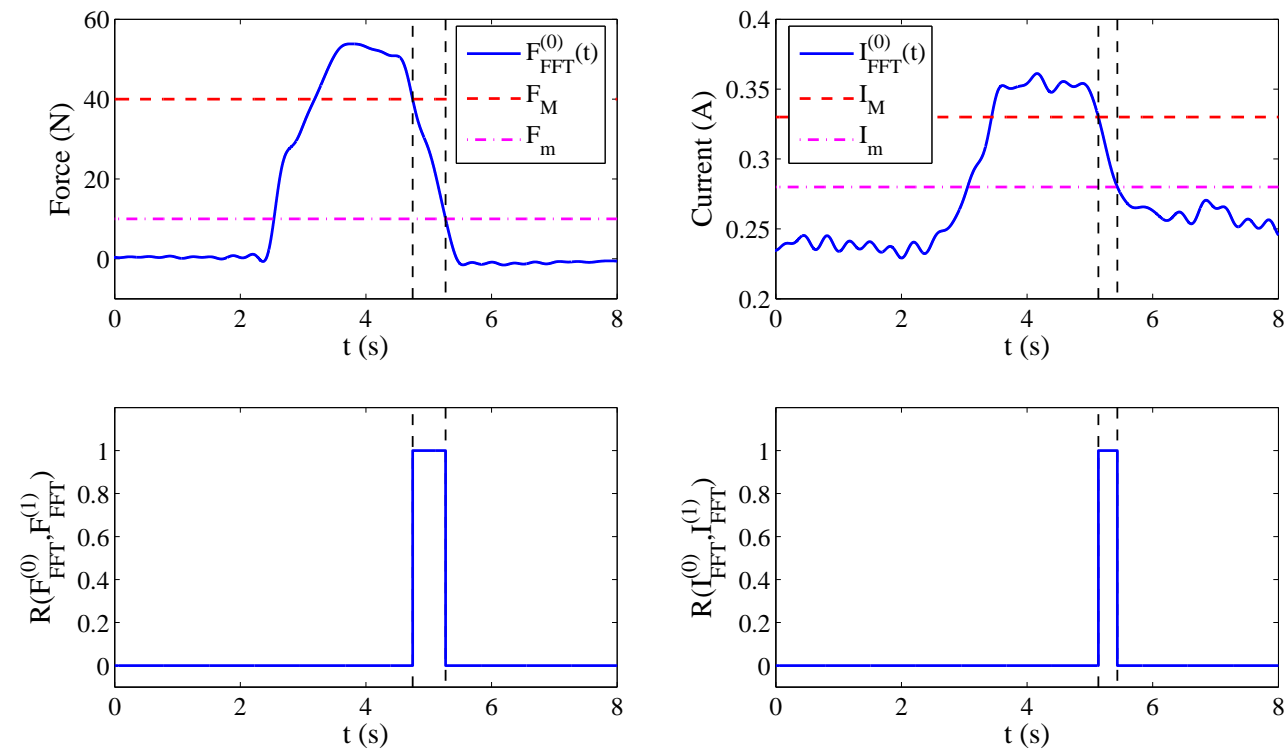

Figure 11: The filtered signals and switching functions for the force and electric current.

Fig. 11 shows the function $R\left(F_{\mathrm{FFT}}^{(0)}(t), F_{\mathrm{FFT}}^{(1)}(t)\right)$ and $R\left(I_{\mathrm{FFT}}^{(0)}(t), I_{\mathrm{FFT}}^{(1)}(t)\right)$. The function value $R\left(u^{(0)}(t), u^{(1)}(t)\right)=1$ means that the force and current decrease in the defined interval between maximal and minimal values, which indicate the exit of the drill from the bone.

So, the function $R\left(u^{(0)}(t), u^{(1)}(t)\right)$, where $u^{(0)}(t)$ and $u^{(1)}(t)$ are estimated on-line, can be used to stop and reverse the drill feed movement in real-time applications, which allows us to successfully remove the drill bit from the bone. The similarity of the on-line function $R\left(u^{(0)}(t), u^{(1)}(t)\right)$ with the ideal off-line function $R\left(u_{\mathrm{FFT}}^{(0)}(t), u_{\mathrm{FFT}}^{(1)}(t)\right)$ will be the measure of the quality of the on-line estimators for the detection of the exit of the drill from the bone.

\subsection{Comparison of the estimation results for $A D$, TD, and $O A D E$}

The proposed AD, TD, and OADE will be used in the estimation of the measured force and current signals, and their first derivatives. Based on these estimations, the switching function will be calculated and then compared with the ideal off-line switching function. The parameters of the algebraic estimator are $a=3$ and $n=5$, the parameter of the TD is $\varepsilon=0.25$, and the reset time of the OADE is $T_{r}=1.5$ and $\epsilon=0.2$.

Fig. 12 shows the estimation of the force $F_{1}^{(0)}(t)$, the estimation of the force first derivative $F_{1}^{(1)}(t)$ and switching function $R\left(F_{1}^{(0)}(t), F_{1}^{(1)}(t)\right)$ by using the proposed AD, TD, and OADE. The low noise attenuation performance in the estimation of the force first derivative for TD and OADE leads to the non-reliable behavior of the switching function. The high frequency chattering-like switching between zero and one values has occurred, even during the time interval when the drill is entering the bone. The proposed AD has a better noise attenuation performance in the estimation of the signal first derivative, which pro- 

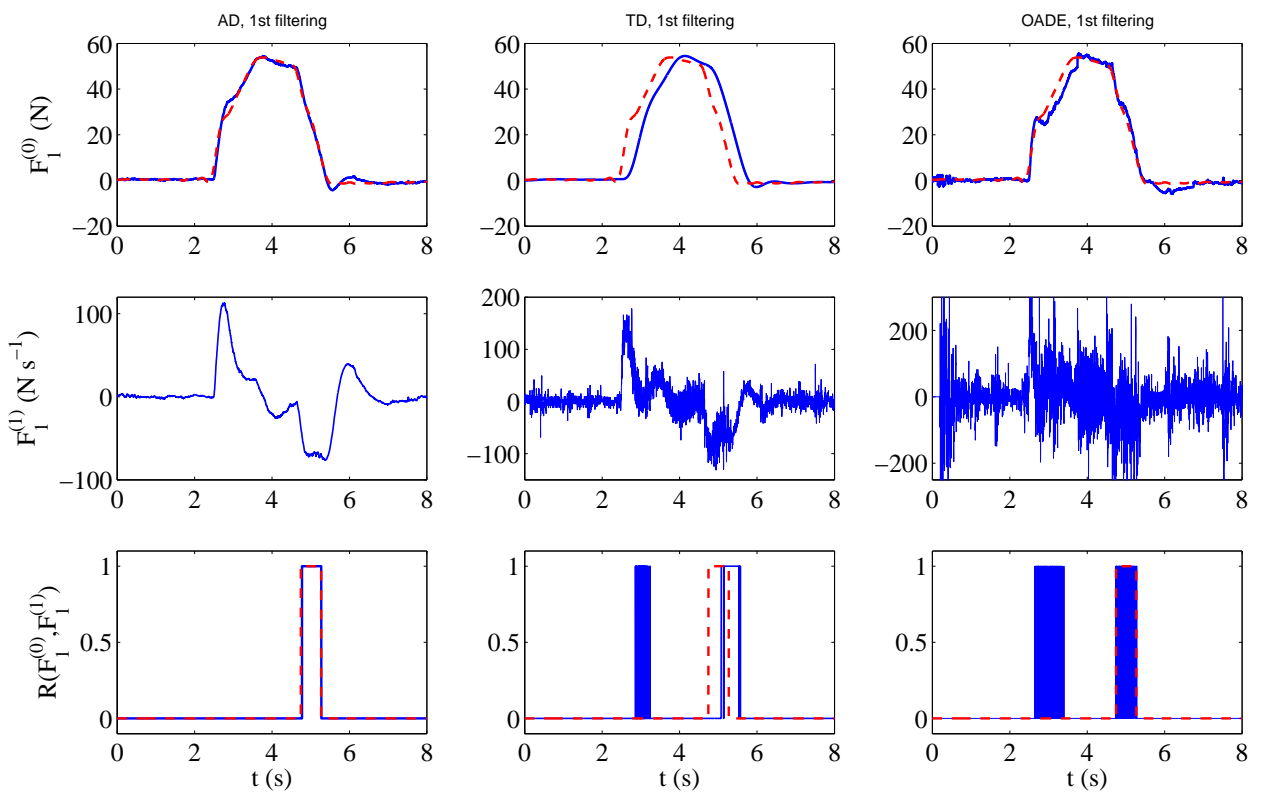

Figure 12: The first filtering estimation results for the force signal $F_{1}^{(0)}(t)$ and its derivative $F_{1}^{(1)}(t)$ for the proposed AD, TD [13] and OADE [17]. The switching function $R\left(F_{1}^{(0)}(t), F_{1}^{(1)}(t)\right)$ (full line) is compared with the ideal switching function $R\left(F_{\mathrm{FFT}}^{(0)}(t), F_{\mathrm{FFT}}^{(1)}(t)\right)$ (dashed line).

vides a reliable switching function, and is almost the same as an ideal switching function $R\left(F_{\mathrm{FFT}}^{(0)}(t), F_{\mathrm{FFT}}^{(1)}(t)\right)$.

Fig. 13 shows the force estimation results in the case of the second filtering, for the proposed AD, TD and OADE. As explained in Section 4.3, the second filtering means that the filtered signal $F_{1}^{(0)}(t)$ is used as the input of the another filter with the same structure. The outputs of the second filter are the estimated force $F_{2}^{(0)}(t)$ and its derivative $F_{2}^{(1)}(t)$. As was expected, additional filtering provides better noise attenuation in the case of the first derivative estimation $F_{2}^{(1)}(t)$ but it also causes a larger error of the estimated signal $F_{2}^{(0)}(t)$. Consequently, the best performance of the switching function can be obtained by taking the first filtering signal estimate $F_{1}^{(0)}(t)$ and the second filtering derivative estimate $F_{2}^{(1)}(t)$ as arguments.

In the case of the TD, the chattering of the switching function is completely eliminated and there is no switching during the interval when the drill is entering the bone. However, the switching function has a time delay that similar to the time delay of the estimated signal $F_{1}^{(0)}(t)$ for TD in Fig. 12. In the case of the OADE, the chattering of the switching function is reduced but switching still appears in the interval when drill is entering the bone. The switching function of the proposed AD is the same as that in the case of the first filtering.

Fig. 14 and Fig. 15 show the results of the first and second filtering in the case of the estimation of the electric current and its time derivative. Because the electric current signal is more noisy than the force signal, it is especially reflected on the estimation of the signal 

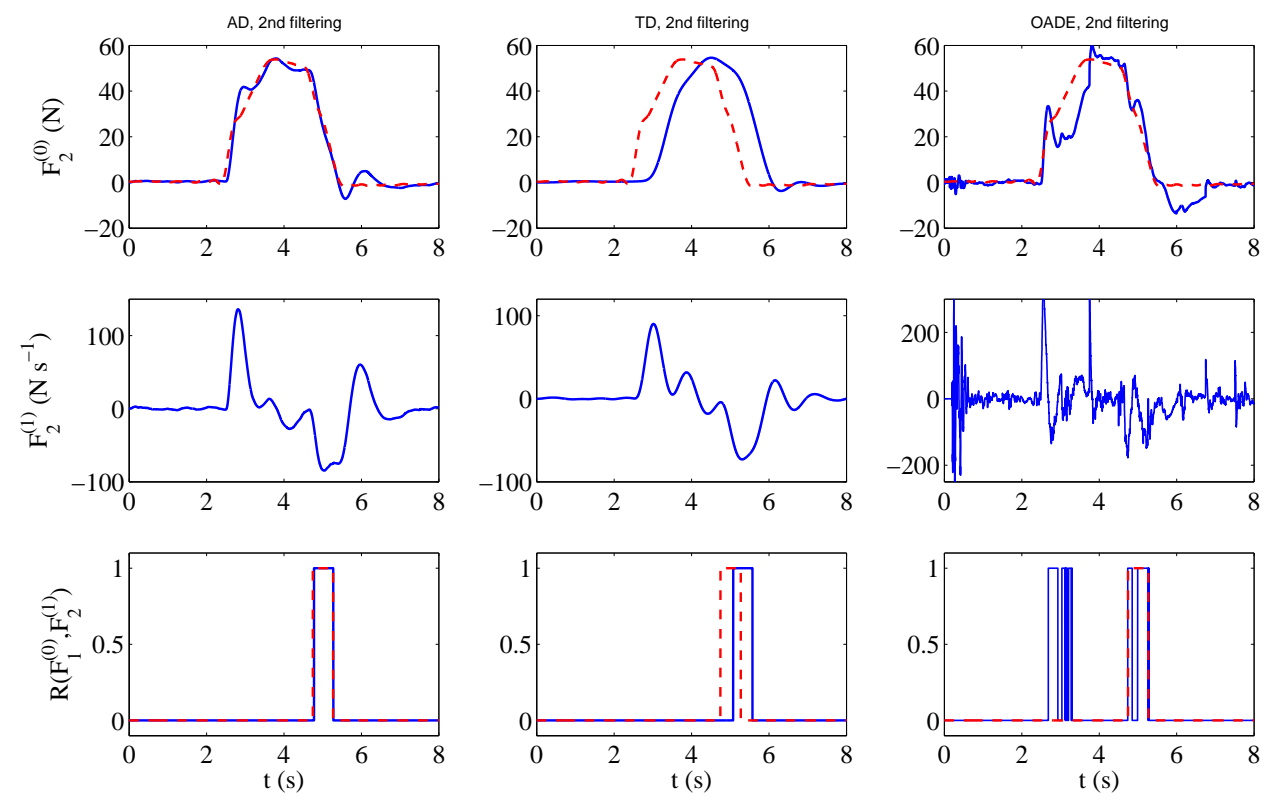

Figure 13: The second filtering estimation results for the force signal $F_{2}^{(0)}(t)$ and its derivative $F_{2}^{(1)}(t)$ for the proposed AD, TD [13] and OADE [17]. The switching function $R\left(F_{1}^{(0)}(t), F_{2}^{(1)}(t)\right)$ (full line) is compared with the ideal switching function $R\left(F_{\mathrm{FFT}}^{(0)}(t), F_{\mathrm{FFT}}^{(1)}(t)\right)$ (dashed line).

derivative in the case of the TD and OADE. The second filtering improved the estimation of the signal derivative in the case of the TD but the switching function delay was too large to prevent reliable detection of the drill's exit from the bone. In the case of the OADE, the estimation of the signal derivative is not improved after the second filtering and the chattering of the switching function still appears. The proposed AD, similarly to the case of the force signal estimation, provides a reliable switching function, which is almost the same as the ideal switching function $R\left(I_{\mathrm{FFT}}^{(0)}(t), I_{\mathrm{FFT}}^{(1)}(t)\right)$.

\subsection{Discussion of the estimation results with experimental measurements}

The main reason why the TD and OADE exhibit poor noise attenuation performance in the case of the signal derivative estimation is the explicit appearance of the measured noisy signal in the filter output equations (75) and (77), respectively, which is in contrast to the proposed $\mathrm{AD}$ where the filter output equation (72) contains only filter state variables.

In the case of the TD, the first derivative estimate contains the term with the signal measurement noise multiplied with the gain $3 / \varepsilon$. By increasing the value of the parameter $\varepsilon$, the noise influence will be reduced but the signal delay will also be increased.

Similarly, in the case of OADE, the first derivative estimate contains the term with the signal measurement noise multiplied with the gain $42 / t_{i}$, where $t_{i} \in\left[\epsilon, T_{r}\right)$. By simultaneously increasing the value of the parameters $\epsilon$ and $T_{r}$, the noise influence will be reduced but the accuracy of the signal estimation will be decreased. Furthermore, the parameter 

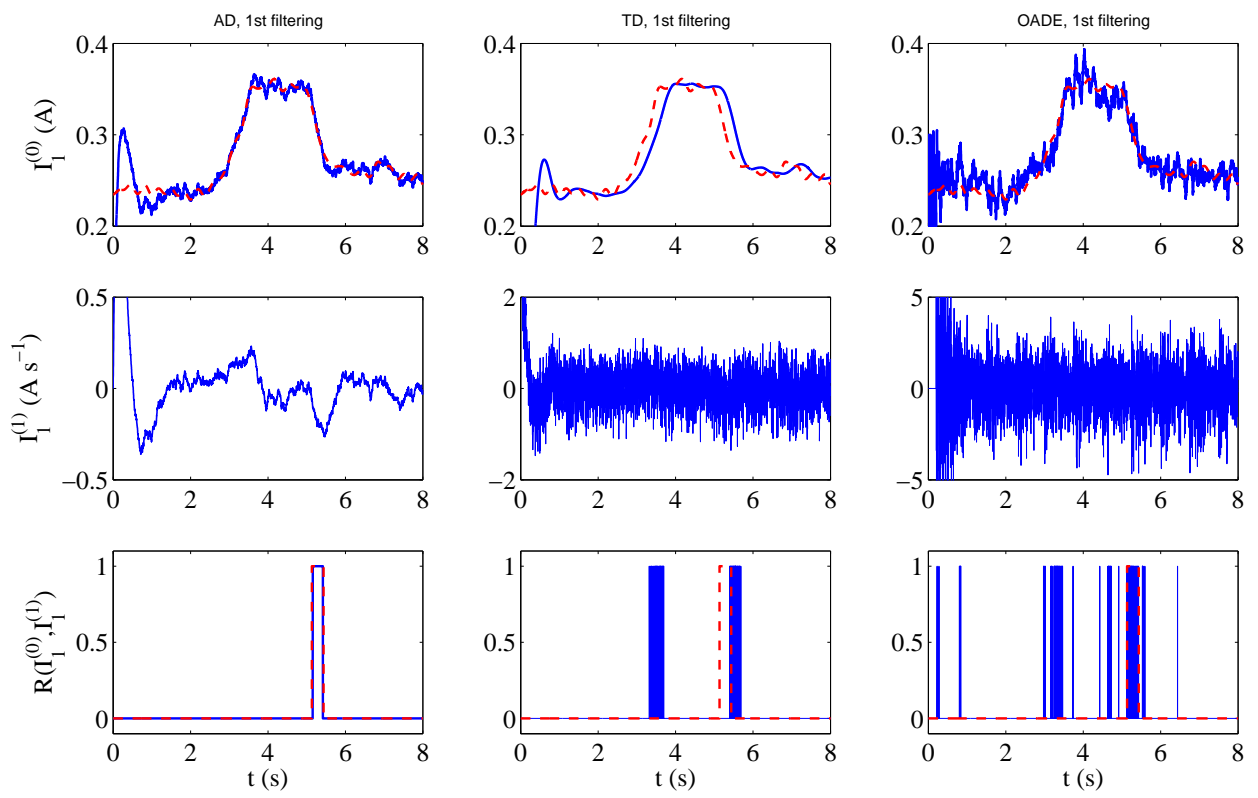

Figure 14: The first filtering estimation results for the current signal $I_{1}^{(0)}(t)$ and its derivative $I_{1}^{(1)}(t)$ for the proposed AD, TD [13] and OADE [17]. The switching function $R\left(I_{1}^{(0)}(t), I_{1}^{(1)}(t)\right)$ (full line) is compared with the ideal switching function $R\left(I_{\mathrm{FFT}}^{(0)}(t), I_{\mathrm{FFT}}^{(1)}(t)\right)$ (dashed line).

$T_{r}$ cannot be arbitrarily increased because of the overflow of the unstable filter (79) state variables.

The estimation results illustrate the high performances of the proposed AD. The main advantages can be summarized as follows: a) high attenuation of the noise effects in the case of derivatives estimation, in comparison with the TD and OADE; b) there is no substantial delay in the filtered signal, in comparison with TD; c) easy implementation in the form of stable continuous linear time-invariant state-space system, in comparison with the OADE; and d) relatively straightforward and less sensitive tuning of filter parameters, in comparison with the TD and OADE.

\section{Conclusions}

This paper presents a new algebraic approach to the estimation of the signal derivatives. The proposed linear estimator provides a stable on-line estimation of the arbitrary number of the higher-order signal derivatives. The estimator has only two tuning parameters: the dimension of the filter and a real stable multiple-pole in the complex domain. These parameters can be easily tuned to provide a good trade-off between estimation accuracy and measurement noise attenuation. Our future work will be oriented towards improvement of the transient performances and application of the proposed estimator for the output tracking problem in feedback control systems. 

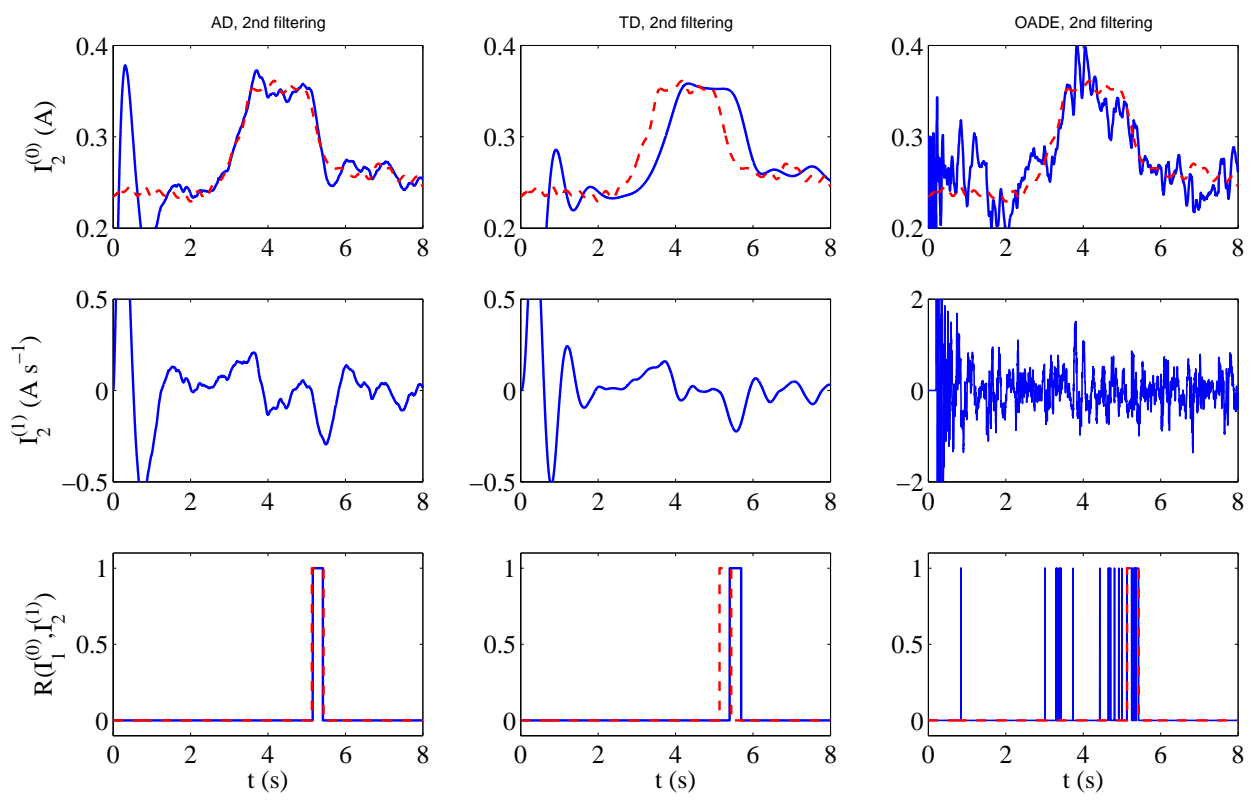

Figure 15: The second filtering estimation results for the current signal $I_{2}^{(0)}(t)$ and its derivative $I_{2}^{(1)}(t)$ for the proposed AD, TD [13] and OADE [17]. The switching function $R\left(I_{1}^{(0)}(t), I_{2}^{(1)}(t)\right)$ (full line) is compared with the ideal switching function $R\left(I_{\mathrm{FFT}}^{(0)}(t), I_{\mathrm{FFT}}^{(1)}(t)\right)$ (dashed line).

\section{Acknowledgment}

This work has been supported by the Croatian Science Foundation under project number IP-09-2014-9870.

\section{References}

[1] H. Lanshammar, On practical evaluation of differentiation techniques for human gait analysis, Journal of Biomechanics 15 (2) (1982) 99-105.

[2] C. Alwi, Halim; Edwards, An adaptive sliding mode differentiator for actuator oscillatory failure case reconstruction, Automatica 49 (2) (2013) 642-651.

[3] P. Soo-Chang, S. Jong-Jy, Design of FIR Hilbert transformers and differentiators by eigenfilter, IEEE Transactions on Circuits and Systems 35 (11) (1988) 1457-1461.

[4] X. Shao, H. Wang, Back-stepping robust trajectory linearization control for hypersonic reentry vehicle via novel tracking differentiator, Journal of the Franklin Institute 353 (9) (2016) 1957-1984.

[5] X. Bu, X. Wu, R. Zhang, Z. Ma, J. Huang, Tracking differentiator design for the robust backstepping control of a flexible air-breathing hypersonic vehicle, Journal of the Franklin Institute 352 (4) (2015) 1739-1765.

[6] A. T. Bahill, J. S. Kallman, J. E. Lieberman, Frequency limitations of the two-point central difference differentiation algorithm, Biological Cybernetics 45 (1) (1982) 1-4.

[7] J. Cullum, Numerical differentiation and regularization, SIAM Journal on Numerical Analysis 8 (2) (1971) 254-265.

[8] R. E. Kalman, R. S. Bucy, New results in linear filtering and prediction theory, Journal of Basic Engineering 83 (1) (1961) 95-108. 
[9] F. Esfandiari, H. K. Khalil, Output feedback stabilization of fully linearizable systems, International Journal of Control 56 (5) (1992) 1007-1037.

[10] Y. Chitour, Time-varying high-gain observers for numerical differentiation, IEEE Transactions on Automatic Control 47 (9) (2002) 1565-1569.

[11] A. Levant, Robust exact differentiation via sliding mode technique, Automatica 34 (3) (1998) 379-384.

[12] A. Levant, Higher-order sliding modes, differentiation and output-feedback control, International Journal of Control 76 (9-10) (2003) 924-941.

[13] H. Feng, S. Li, A tracking differentiator based on Taylor expansion, Applied Mathematics Letters 26 (7) (2013) 735-740.

[14] Z. G. Li, Z. H. Ma, A new approach for filtering and derivative estimation of noisy signals, Circuits, Systems, and Signal Processing 33 (2) (2014) 589-598.

[15] X.-Y. Lu, K. J. Hedrick, Inverse Taylor series problem in linear filtering and related conjectures, in: Proc. of the 2001 American Control Conference, Arlington, VA, 2001, pp. 2528-2529.

[16] M. Fliess, H. Sira-Ramirez, Control via state estimations of some nonlinear systems, in: Proc. of the IFAC Symposium on Nonlinear Control Systtems, Stuttgart, Germany, 2004, pp. 2528-2529.

[17] R. Morales, E. Segura, J. Somolinos, L. Núñez, H. Sira-Ramírez, Online signal filtering based on the algebraic method and its experimental validation, Mechanical Systems and Signal Processing 66-67 (2016) 374-387.

[18] K. D. Listmann, Z. Zhao, A comparison of methods for higher-order numerical differentiation, in: Proc. of the European Control Conference, Zürich, Switzerland, 2013, pp. 9152-9158.

[19] V. Judalet, S. Glaser, C. Boussard, S. Mammar, Implementation of first order algebraic estimators for numerical filtering and derivation applications, IFAC Proceedings Volumes 47 (3) (2014) 9152-9158.

[20] W. Hongwei, W. Heping, A comparison study of advanced tracking differentiator design techniques, Procedia Engineering 99 (2015) 1005-1013.

[21] J. Kasac, T. Zilic, V. Milic, A. Jokic, Frequency-shifting-based stable on-line algebraic parameter identification of linear systems, Journal of the Franklin Institute, available online 22 March 2017, https://doi.org/10.1016/j.jfranklin.2017.03.008.

[22] H. Sira-Ramírez, C. G. Rodríguez, J. C. Romero, A. L. Juárez, Algebraic Identification and Estimation Methods in Feedback Control Systems, John Wiley \& Sons, 2014.

[23] A. Prosperetti, Advanced Mathematics for Applications, Cambridge University Press, 2011.

[24] R. F. Hoskins, Delta Functions: Introduction to Generalised Functions, Woodhead Publishing, 2009.

[25] K. Lundberg, H. Miller, R. Trumper, Initial conditions, generalized functions, and the Laplace transform, IEEE Control Systems Magazine 27 (1) (2007) 22-35.

[26] P. A. Roebuck, S. Barnett, A survey of Toeplitz and related matrices, International Journal of Systems Science 9 (8) (1978) 921-934.

[27] T. Mita, On zeros and responses of linear regulators and linear observers, IEEE Transactions on Automatic Control 22 (3) (1977) 423-428.

[28] H. Kimura, A new approach to the perfect regulation and the bounded peaking in linear multivariable control systems, IEEE Transactions on Automatic Control 26 (1) (1981) 253-270.

[29] J. Reger, M. Fliess, H. Sira-Ramirez, On non-asymptotic observation of nonlinear systems, in: Proc. of the 44th IEEE Conference on Decision and Control, Seville, Spain, 2005.

[30] V. Kotev, G. Boiadjiev, H. Kawasaki, T. Mouri, K. Delchev, T. Boiadjiev, Design of a hand-held robotized module for bone drilling and cutting in orthopedic surgery, in: Proc. of the IEEE/SICE International Symposium on System Integration, Fukuoka, Japan, 2012, pp. 504-509.

[31] T. Staroveski, D. Brezak, T. Udiljak, Drill wear monitoring in cortical bone drilling, Medical engineering \& physics 37 (6) (2015) 560-566. 